\title{
A DEEP PROPER-MOTION SURVEY IN KAPTEYN SELECTED AREAS. I. SURVEY DESCRIPTION AND FIRST RESULTS FOR STARS IN THE TIDAL TAIL OF SAGITTARIUS AND IN THE MONOCEROS RING
}

\author{
Dana I. Casetti-Dinescu, ${ }^{1,2}$ Steven R. Majewski, ${ }^{3}$ Terrence M. Girard, ${ }^{1}$ Jeffrey L. Carlin, ${ }^{3}$ \\ William F. van Altena, ${ }^{1}$ Richard J. Patterson, ${ }^{3}$ and David R. Law ${ }^{4}$ \\ Received 2006 May 31; accepted 2006 August 3
}

\begin{abstract}
We describe a high-precision, deep (to $V \sim 19-21$ ), absolute proper-motion survey that samples $\sim 50$ lines of sight in the Kapteyn selected areas along declination zones $-15^{\circ}, 0^{\circ}$, and $15^{\circ}$. In many fields the astrometric baseline reaches nearly a century. We demonstrate that these data provide typical per star precisions between $\sim 1$ and 3 mas $\mathrm{yr}^{-1}$ to the above magnitude limits, with the absolute reference frame established by numerous extragalactic sources in each survey field. Combined with existing and ongoing photometric and radial velocity surveys in these fields, these astrometric data will enable, among other things, accurate detailed dynamical modeling of satellite interactions with our Galaxy. In this contribution we describe the astrometric part of our survey and show preliminary results along the trailing tail of the Sagittarius dwarf galaxy, and in the Monoceros Ring region.
\end{abstract}

Key words: astrometry — Galaxy: kinematics and dynamics — Galaxy: structure — surveys

\section{INTRODUCTION}

Modern, deep, uniform, large-area photometric surveys have shown unambiguously that the Milky Way outer halo contains accretion-derived substructure (e.g., Ibata et al. 2001; Newberg et al. 2002; Majewski et al. 2003; Rocha-Pinto et al. 2003, 2004; Vivas et al. 2004; Conn et al. 2005a). These structures of known or assumed remnants of satellite accretion have long-lived, coherent tidal features that can be used to model the Galactic gravitational potential (e.g., Ibata et al. 2001; Law et al. 2005), as well as the characteristics of the original satellites (e.g., Johnston et al. 1999). However, such modeling studies have been limited by the meager available kinematical data over large angles along the tidal features. While radial-velocity programs have just recently begun to address this problem for the few known Galactic tidal tails (Sagittarius: Majewski et al. 2004, Vivas et al. 2004; the Monoceros Ring: Crane et al. 2003), no systematic survey has begun to address the transverse (tangential) velocities (i.e., absolute proper motions). Without this information, dynamical models remain poorly constrained, and therefore limited to describing merely a range of possible events rather than an accurate description of the real event.

The work described here is a proper-motion survey that provides high-precision (1-3 mas $\mathrm{yr}^{-1}$ per well-measured star) absolute and relative proper motions down to a magnitude of $V \sim 19$, and for a few selected fields down to $V \sim 21$, in $\sim 50$ lines of sight in the selected areas (SAs) designed by Kapteyn for Galactic structure studies in 1906. Current proper-motion surveys do not achieve this precision at a similar magnitude limit

\footnotetext{
1 Astronomy Department, Yale University, P.O. Box 208101, New Haven, CT 06520-8101; dana@astro.yale.edu, girard@astro.yale.edu, vanalten@astro .yale.edu.

2 Astronomical Institute of the Romanian Academy, Strada Cutitul de Argint 5, RO-75212 Bucharest 28, Romania.

${ }^{3}$ Department of Astronomy, University of Virginia, P.O. Box 400325, Charlottesville, VA 22904-4325; srm4n@mail.astro.virginia.edu, jc4qn@mail .astro.virginia.edu, rjp01@mail.astro.virginia.edu.

${ }^{4}$ Department of Astronomy, Mail Code 105-24, California Institute of Technology, 1200 East California Boulevard, Pasadena, CA 91125; drlaw@astro .caltech.edu.
}

and thus have limited capability of detecting and characterizing distant halo substructure. To date there are only a few similarly deep, precise, pencil-beam-type proper-motion data sets that are primarily centered on globular clusters or dwarf spheroidal galaxies, and even fewer that are focused on Galactic field stars (e.g., Chiu 1980; Majewski 1992; Guo et al. 1993; Dinescu et al. 2002). Obviously, ground-based studies of this type are limited to parts of the sky where suitable first-epoch astrometric data exist. Here we exploit a unique cache of nearly century old, deep photographic plates having good scale, as well as other plate material collected in the Kapteyn fields.

We intend to complement the new proper-motion data with radial velocities, distances, and metallicity estimates from our own photometric and spectroscopic work, as well as from overlapping surveys such as the Two Micron All Sky Survey (2MASS), the Sloan Digital Sky Survey (SDSS), the Quasar Equatorial Survey Team (QUEST), and the Radial Velocity Experiment.

With this survey we aim to (1) determine the extent and orbital motion of the highly obscured Monoceros Ring-like structure above and below the Galactic plane, and explore its possible relation to other low-latitude structures, such as the Canis Major overdensity, (2) characterize the transverse motion of the Sagittarius tidal streams, (3) search for and characterize additional substructures in the halo of the Milky Way, and (4) determine the kinematical properties of numerous thick-disk and halo stars in our fields as a function of Galactic position.

Future papers will include detailed kinematical analyses for specific regions, while in this paper we characterize the survey and show its potential in two specific regions where tidal streams have already been identified from other sources. In $\S 2$ we describe the survey in detail. In $\S 3$ we show results in a few SA fields, and a brief summary is presented in $\S 4$.

\section{SURVEY DESCRIPTION}

\subsection{The Collection of Photographic Plates}

This survey is made possible by the visionary, now centuryold Milky Way survey introduced by Kapteyn in 1906. Kapteyn devised the Plan of Selected Areas as a means to systematically study the Milky Way. The plan, as originally envisioned, involved 


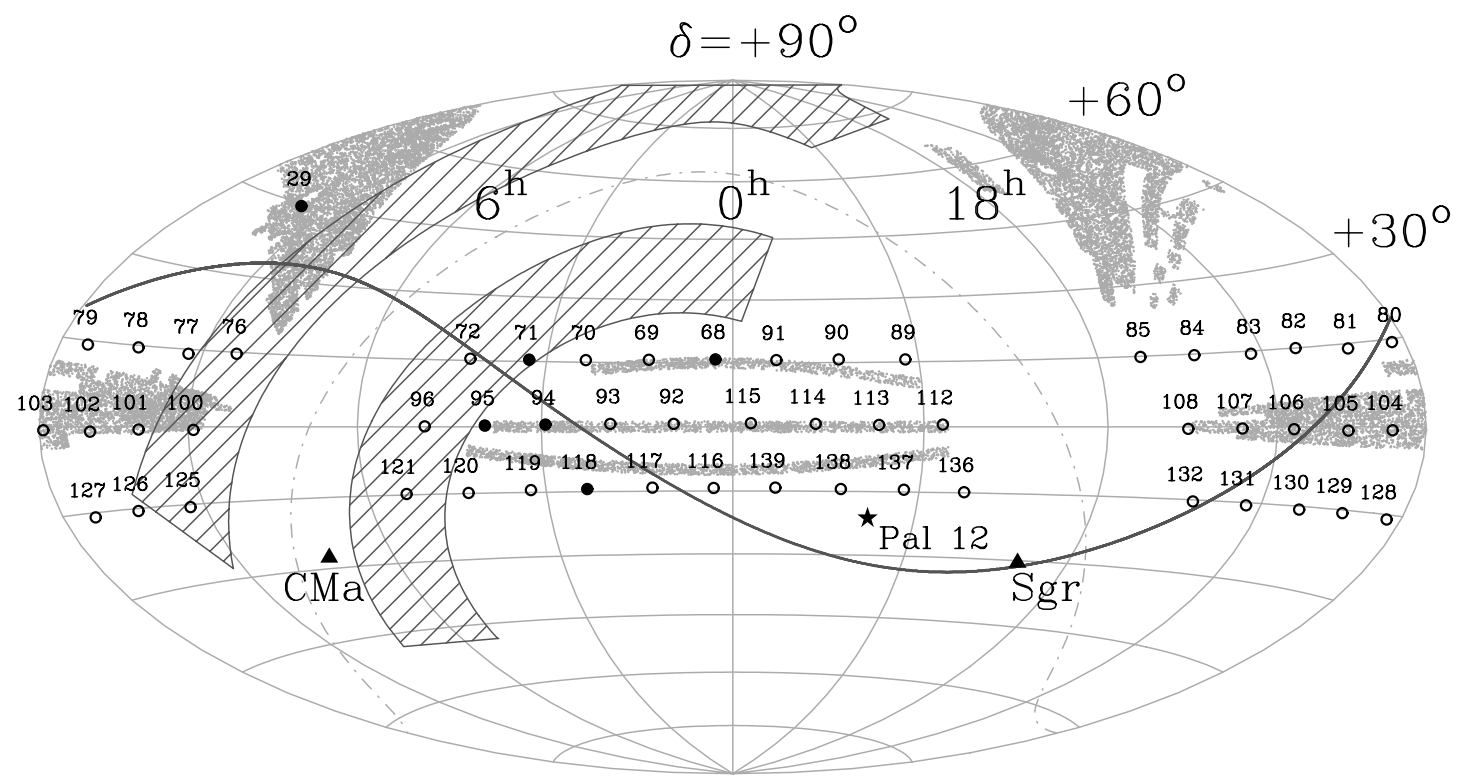

FIG. 1.-Distribution of the SA field centers (open circles) in equatorial coordinates. Filled circles show the fields that have additional $4 \mathrm{~m}$ plates. The solid line shows the approximate orbital plane of Sgr, and the dash-dotted line shows the Galactic plane. The gray areas show the SDSS coverage as given by the catalog of QSOs (Schneider et al. 2005). The cross-hatched bands represent the areas where the Mon structure was mapped. The globular cluster Pal 12, Sagittarius's core, and the CMa dwarf galaxy candidate are also marked.

photometry, astrometry, and spectroscopy of stars in 206 SAs collected at numerous observatories around the world and focused on characterizing the "sidereal world"; the perceived importance of this grand effort in the early 20th century prompted the creation of International Astronomical Union (IAU) Commission 32: Selected Areas, as well as the Subcommittee on Selected Areas of IAU Commission 33: Structure and Dynamics of the Galactic System. Among the important early contributions to the SA program was the Carnegie Institution's systematic analysis of stellar photometry from Mount Wilson 60 inch $(1.5 \mathrm{~m})$ plates of the 139 northern accessible SAs by Seares et al. (1930). The photographic plates used in this analysis were taken by E. Fath and H. Babcock with the 60 inch telescope between 1909 and 1912. For 54 near-equatorial fields there exist deliberately matched (in area, approximate plate scale, and depth) photographic plates taken with the Las Campanas du Pont $2.5 \mathrm{~m}$ telescope by S. Majewski between 1996 and 1998. This collection of photographic plates provides the opportunity for an unprecedentedly deep, high-precision proper-motion survey that takes advantage of the excellent plate scale $\left(10^{\prime \prime} .92 \mathrm{~mm}^{-1}\right.$ for the $2.5 \mathrm{~m}$ du Pont telescope and 27.'12 $\mathrm{mm}^{-1}$ for the 60 inch Mount Wilson telescope) of the images taken in both epochs, which span a $\sim 90 \mathrm{yr}$ baseline. Each field of view is $40^{\prime} \times 40^{\prime}$. Unfortunately, because the old 60 inch plates go only as deep as $V \sim 19$ for blue objects, there are very few galaxies to determine the correction to absolute proper motion in a $40^{\prime} \times 40^{\prime}$ field of view, with those that are available being at the plate limiting magnitude, and therefore yielding rather poor centroids. Primarily for this reason, we have included in our proper-motion determinations the first Palomar Observatory Sky Survey (POSS-I) plates as measured by both the Space Telescope Science Institute (STScI; the Digitized Sky Survey [DSS]) and the US Naval Observatory (USNO). The POSS-I plates were taken in the early 1950s with the Oschin Schmidt telescope and have a plate scale of $67.2 \mathrm{~mm}^{-1}$. While the scale and the digitization of the POSS-I plates (see $\S 2.3$ ) are much poorer than those of the other two sets of plates, they offer a $40 \mathrm{yr}$ baseline with the du Pont plates and extend the proper-motion limiting magnitude to $V \sim 20-21$.
Minimally, each field has an early-epoch Mount Wilson plate with two offset images, one from a 60 minute exposure and one from a 3 minute exposure. Many fields have a second early Mount Wilson plate, sometimes with two exposures, sometimes with one. For each field there are two recent-epoch du Pont plates taken in the blue (IIIa-J + GG385) and visual (IIIa-F + GG495) passbands. These plates also contain a pair of offset exposures of about 60 and 3 minute integration. The intermediate-epoch POSS-I plates were taken in the blue (103a-O, no filter) and red (103a-E + RP2444) passbands, with typical exposure times of 10 and 50 minutes, respectively.

Finally, a handful of fields have Kitt Peak National Observatory Mayall $4 \mathrm{~m}$ prime focus photographic plates (plate scale $18 . " 6 \mathrm{~mm}^{-1}$ ) taken in the mid-1970s by A. Sandage and mid1990 s by S. Majewski. The modern plates were taken in the blue (IIIa-J + GG385) and visual (IIIa-F + GG495) passbands, while the Sandage plates were taken in the blue (IIa-O + GG385/GG3), visual (IIa-D + GG495/GG11), and red (127-04 + RG610) passbands.

\subsection{Area Coverage}

In Figure 1 we show the location of the centers of the SA fields (open circles) on the sky in an Aitoff projection. The filled circles represent the fields that also have Mayall $4 \mathrm{~m}$ plates. The survey samples three declination zones $\left(0^{\circ}\right.$ and $\left.\pm 15^{\circ}\right)$ and the full range in right ascension, except for low Galactic latitude $\left(|b|<25^{\circ}\right)$ zones. The Galactic plane is represented with a gray dash-dotted line. There is one field, SA 29, that is at higher declination and that has only $4 \mathrm{~m}$ plates. We are including this field because it falls within the QSO catalog determined from Data Release 3 (DR3) of the SDSS area (Fig. 1, gray area). Although, for our astrometric reductions, we make use of the photometry from DR4, Figure 1 highlights the approximate SDSS footprint corresponding to the QSO catalog. Thus, it can be seen that for a good number offields QSOs have already been identified that can be used in setting the absolute proper-motion reference frame.

We have indicated the location of the Sagittarius dwarf galaxy's (Sgr) center and that of the globular cluster Pal 12, which is 
believed to have belonged to Sgr according to proper-motion data (Dinescu et al. 2000), surrounding field photometry (MartínezDelgado et al. 2002), and chemical abundances (Cohen 2004). The solid dark line shows Sgr's most recent orbit determination (Dinescu et al. 2005a). The orbit is roughly indicative of Sgr's tidal streams, which can be as wide as $10^{\circ}$, as Pal 12's location, for instance, suggests. It is apparent that Sgr's southern, trailing arm, which is closer to the Sun $(\sim 20-30 \mathrm{kpc}$; Majewski et al. 2003) than much of the northern, leading arm, will be sampled in perhaps six SA fields (depending on the true stream width) from R.A. $=0^{\mathrm{h}}$ to $5^{\mathrm{h}}$. In this paper we present results for SAs 93 and 94.

The second most well-known putative halo substructure is the Monoceros Stream or Ring (Newberg et al. 2002; Ibata et al. 2003), a low Galactic latitude structure that is represented here by the two cross-hatched bands above and below the Galactic plane. This area is drawn only approximately as based on observations from Yanny et al. (2003), Ibata et al. (2003), RochaPinto et al. (2003), Crane et al. (2003), Conn et al. (2005a), and Martin et al. (2006). The stellar overdensity discovered by Martin et al. (2004) in the constellation of CMa and subsequently analyzed by other groups may be a distinct structure (but cf. RochaPinto et al. 2006), and it has been suggested to be the core of the satellite that is responsible for the Monoceros Stream (see, e.g., Peñarrubia et al. 2005). We have marked the location of this structure (CMa), and we note that Conn et al. (2005b) claim, based on radial velocities, to have detected the Monoceros Ring behind the CMa structure as well. At first glance, between 6 and 12 SA fields are likely to sample the Monoceros Ring. Fields SA 71 and 72 are more problematical because both Sgr and Mon are expected in these areas (see Fig. 1). This paper presents results in SAs 96, 100, and 101, which fall within/close to the Mon region.

Another equatorial survey that overlaps some of our propermotion survey is the QUEST survey, which identifies RR Lyrae variable stars along the celestial equator (Vivas et al. 2004). Their most recent results (Duffau et al. 2006) indicate the discovery of a new halo structure in the constellation of Virgo that is not related to either Sgr or Mon (see also Jurić et al. 2006). Two of our SA fields, 103 and 104, are in the area indicated by QUEST to sample the Virgo structure. Provided the depth of the plates allows it, we may be able to determine an absolute proper motion for this structure in the near future.

\subsection{Photographic Plate Measurements}

\subsection{1. du Pont $2.5 \mathrm{~m}$, Mount Wilson 60 inch, and Mayall $4 \mathrm{~m}$ Scans}

For each field we start by fully digitizing one du Pont, one 60 inch, and one $4 \mathrm{~m}$ plate. These initial coarse scans serve to build input lists for high-resolution scans. All of the scans, except for those of the Mayall 4 m plates in SAs 29, 71, 94, and 118, were done with the Yale PDS microdensitometer. The coarse scans of most of the $4 \mathrm{~m}$ plates were done with the University of Virginia's microdensitometer. The size of the field is defined by the 10 inch $\times 10$ inch du Pont plate size, which corresponds to $40^{\prime} \times 40^{\prime}$. For the 60 inch plate in each field, we digitize the same size area that matches the du Pont field. This corresponds to a 10 $\mathrm{cm}$ box located at the center of the Mount Wilson plate. Stellar images on the 60 inch plates are affected by coma, and outside this region they are practically unusable for astrometric purposes. Based on these coarse scans, an input list of objects is determined using the software package SExtractor (Bertin \& Arnouts 1996) for each epoch. Long and short exposures on each plate are separated into two different lists. Then, each exposure on each plate is measured in a fine raster, object-by-object mode, with a pixel size of $12.7 \mu \mathrm{m}(0.138)$ for the du Pont plates and $10 \mu \mathrm{m}$ $(0$ "'275) for the 60 inch plates. The input lists for the $4 \mathrm{~m}$ plates were made from the coarse scans done at the University of Virginia, using the software package FOCAS (Valdes 1982). ${ }^{5}$ These $4 \mathrm{~m}$ input catalogs prepared earlier (Dinescu et al. 2002) were used to measure the $4 \mathrm{~m}$ plates at Yale in a fine raster mode with a pixel size of $10 \mu \mathrm{m}\left(00^{\prime \prime} 186\right)$. The objects' positions, instrumental magnitudes, and other parameters were derived from the fine raster scans using the Yale two-dimensional Gaussian centering routines (Lee \& van Altena 1983). As is customary, a set of five to eight stars well distributed over the plate are repeatedly measured during the scan in order to monitor and correct for thermal drifts during the scan.

From coordinate transformations of same-epoch, same-telescope plates we obtain, for well-measured stars, a centering precision of $1.2 \mu \mathrm{m}$ (13 mas) per single measurement per star for the du Pont plates. For the 60 inch plates this number is $3.3 \mu \mathrm{m}$ (90 mas), while for the $4 \mathrm{~m}$ plates it is $1.3 \mu \mathrm{m}$ (24 mas).

\subsubsection{POSS-I Scans}

There are two readily available scans of the POSS-I plates: those of the red plates done with a PDS machine at STScI, widely known as the DSS, and those of both blue and red plates done at the USNO Flagstaff Station with the Precision Measuring Machine (PMM; see Monet et al. 2003 for its description). The DSS scans are retrieved directly from the Web, ${ }^{6}$ as the area in question is smaller than a degree on a side. The resolution of the DSS scans is $25 \mu \mathrm{m}$ pixel ${ }^{-1}\left(1^{\prime \prime} 7\right.$ pixel $\left.^{-1}\right)$.

Sections of the PMM scans were kindly made available to us by S. Levine at USNO, Flagstaff. These scan sections are centered on the SA fields and cover $40^{\prime} \times 40^{\prime}$. The PMM is an 8 bit, fast measuring machine that uses a CCD detector to take "footprint" images of the photographic plate. The POSS-I plates were scanned at a resolution of $13 \mu \mathrm{m}$ pixel $^{-1}\left(0.9\right.$ pixel $\left.^{-1}\right)$. Each Schmidt plate is covered by some 588 exposures with a field of view of $20^{\prime} \times 15^{\prime}$ each. The CCD footprints are assembled together in the subsequent software by using an offset, to provide the entire digitized sky (see details in Monet et al. 2003). The "stitching" of the CCD footprints, however, is not perfect, and thus positiondependent systematics are introduced (see $\S 2.5$ ). Without access to the individual PMM footprints, a method is required to correct the systematics in the assembled scans. We have made use of the DSS scans, which were produced by a traditional PDS measuring machine, to correct the PMM positions (see $\S 2.5$ ). The SA field scans from DSS and USNO are processed as follows for each POSS-I plate: Objects are detected with SExtractor and then recentered with the Yale centering routines. Coordinate transformations between overlapping plates for the DSS measurements indicate a centering precision of $2.1 \mu \mathrm{m}$ (141 mas) per single measurement per star. Similarly, for the USNO scans we obtain $\sim 2.6 \mu \mathrm{m}$ (174 mas) for both the red and blue plates. Based on these values, if we use only the du Pont and the POSS-I plates (a $\sim 40$ yr baseline), we can obtain a proper-motion uncertainty

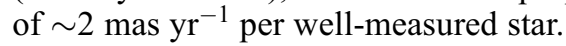

\subsection{Photometry and Spectroscopy}

Here we only briefly mention our campaign to obtain photometry and spectroscopy in the SA fields; more details of these observations will be presented in subsequent papers. All SA fields had $U B V \mathrm{CCD}$ photometry taken with the SITe No. 1

\footnotetext{
${ }^{5}$ See also Valdes's 1993 FOCAS User's Guide, an NOAO document available at ftp://iraf.noao.edu/iraf/docs/focas/focasguide.ps.Z.

${ }^{6}$ See http://archive.stsci.edu/cgi-bin/dss_form.
} 

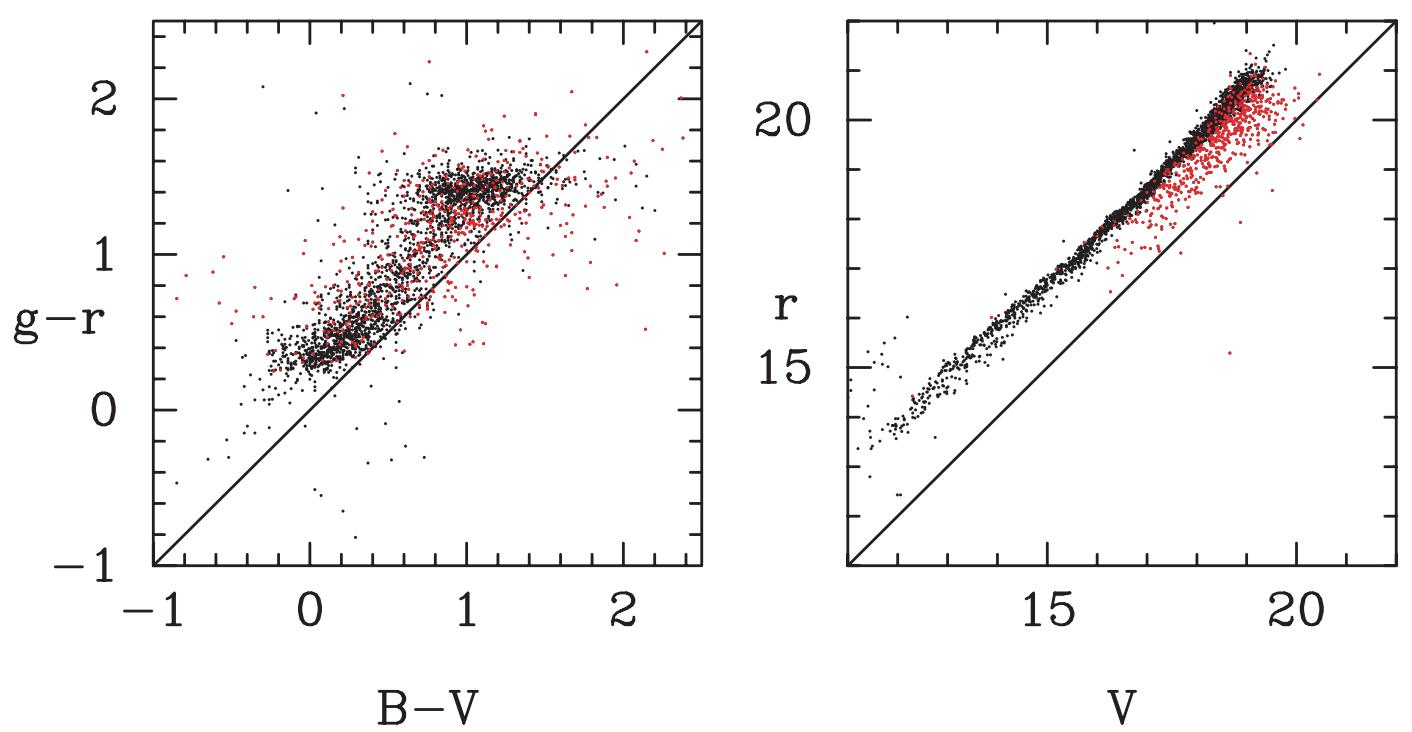

FIG. 2.-SDSS $(g-r)$ colors as a function of photographic $(B-V)$ colors (left) and SDSS $r$ magnitudes as a function of photographic $V$ magnitudes $(r i g h t)$. The photographic magnitudes are derived from the scan of a du Pont plate calibrated to CCD photometry. The $B$ and $V$ magnitudes are not calibrated to the standard Johnson system. Black symbols represent the stars, while red symbols represent the galaxies.

$2048^{2} \mathrm{CCD}$ on the Swope $1 \mathrm{~m}$ at Las Campanas Observatory on the nights of UT 1997 December 23-31, 1998 June 19-26, and 1998 December $15-16$. The CCD field of view covers 30\% $\left(22^{\prime} \times 22^{\prime}, 00^{\prime \prime} 697\right.$ pixel $\left.^{-1}\right)$ of the astrometric field. Exposure times were 120, 200, and $900 \mathrm{~s}$ for the Johnson $V, B$, and $U$ filters, respectively, yielding data shallower by $1-1.5 \mathrm{mag}$ than the typical du Pont plates for red stars, and by $\sim 0.3$ mag for blue stars. Short exposures $(5,7$, and $40-45 \mathrm{~s}$ in $V, B$, and $U$ ) were also taken to obtain photometry for the bright stars in these fields.

For the astrometric reductions we need $B V$ colors for all stars to map out the color terms. These were obtained by calibrating photographic instrumental magnitudes for each blue and visual du Pont plate with CCD magnitudes. The CCD magnitudes used in this process are not calibrated to the standard Johnson system because at the time the astrometric reductions were done, the CCD photometry had not been calibrated yet. Nevertheless, the CCD photometry helped to linearize the photographic magnitudes.

To date, the reduced CCD data cover only a handful of fields. Therefore, for the rest of the fields where there is coverage with SDSS, we have used the SDSS $(g-r)$ colors in the astrometric reductions. In Figure 2 (left) we show the photographic, CCDcalibrated $B-V$ colors versus the SDSS $(g-r)$ colors in SA 100 . Black symbols show stars, and red symbols show galaxies. The good color correlation justifies our use of the $(g-r)$ colors in the astrometry. Figure 2 (right) shows the relationship between the SDSS $r$ magnitude and the photographic $V$ magnitude. This shows that the du Pont plates reach a limiting magnitude of $r \sim 21$.

For the fields where we have detected structure in the propermotion distribution, as well as in the corresponding SDSS colormagnitude diagram (CMD), we have started an observing program with Hydra on the $3.5 \mathrm{~m}$ Wisconsin-Indiana-Yale-NOAO telescope in order to measure radial velocities. So far, preliminary radial velocities have been obtained in SAs 71 and 96. These results will be presented elsewhere.

\subsection{Astrometry}

The du Pont plates were precorrected for differential refraction (third-order refraction theory; Taff 1981, p. 79) and for distortion (see details in Dinescu et al. 2000). The distortion coefficients applied here were determined from the coordinate transformations of eight visual and seven blue plates into the Second USNO CCD Astrograph Catalog (UCAC2; Zacharias et al. 2004). The distortion coefficient for the visual plates is $(-6.55 \pm 0.09) \times$ $10^{-8} \mathrm{~mm}^{-2}$, and for the blue plates it is $(-7.02 \pm 0.06) \times$ $10^{-8} \mathrm{~mm}^{-2}$. Between 100 and 200 stars in common with UCAC2 in the various SA fields were used to model the coordinate transformations. The center of distortion was assumed to coincide with the tangent point. The tangent point was determined by minimizing quadratic terms in the transformations of plate coordinates into the UCAC2 positions (see, e.g., Guo et al. 1993). The cubic distortion coefficients determined here, although slightly smaller than those determined by Cudworth \& Rees (1991), are in agreement within quoted uncertainties. The center of distortion is determined with a precision of $\sim 0.5 \mathrm{~mm}$, or 5."5.

The Mayall $4 \mathrm{~m}$ plates were precorrected for distortion using the coefficients from Chiu (1976). The center of distortion is determined similarly to the process used for the du Pont plates, i.e., by minimizing quadratic terms from a coordinate transformation into UCAC2. This gives a distortion center known to no better than $\sim 0.05 \mathrm{~mm}\left(1^{\prime \prime}\right)$. We have not precorrected the $4 \mathrm{~m}$ plates for differential refraction, as this effect is much smaller than that of distortion; for the du Pont plates these effects are similar in size (Dinescu et al. 2000).

The positions determined from the DSS scans of the POSS-I plates were used to correct the positions determined from the PMM scans, which are affected by the assemblage of multiple CCD footprints (see $\S 2.3$ ). In Figure 3 we show the residuals from a coordinate transformation between PMM measurements and DSS measurements as a function of position. The transformation includes up to fourth-order terms. The top two panels show the residuals for the red plate (i.e., two different measurements for the same red plate: PMM into DSS) and the bottom two for the blue plate (i.e., blue PMM measurements into red DSS measurements) as labeled. The right panels show the residuals after the correction was applied. The correction for each object is derived by taking a local average (of some 20 neighbors) from a two-dimensional map of the residuals shown in Figure 3.

It is well known that astrometry from the wide-field POSS-I plates, which were taken with a Schmidt telescope, is strongly affected by position- and magnitude-dependent systematics (e.g., 

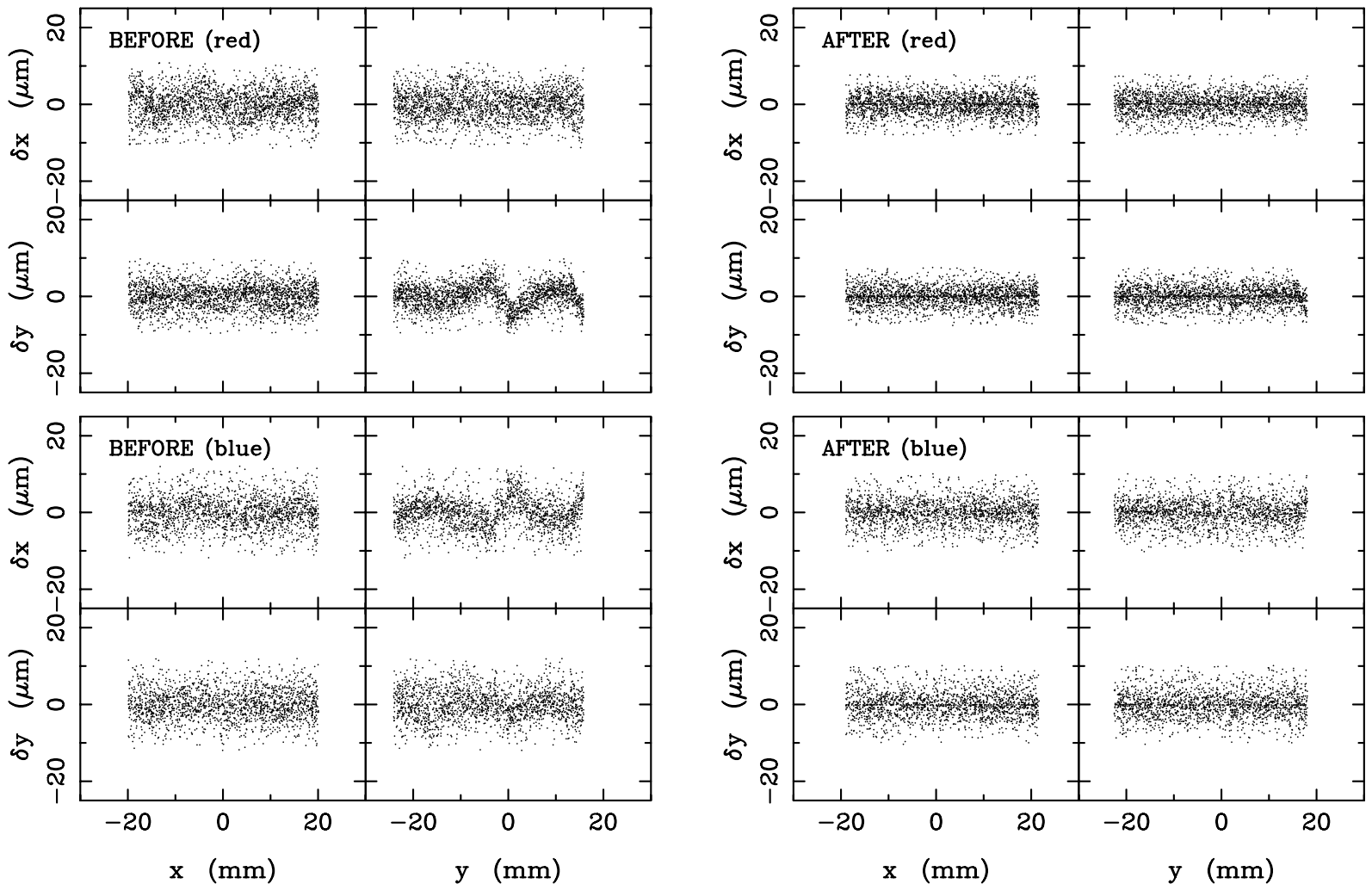

FIG. 3.- Residuals from a transformation of the PMM measurements into the DSS measurements. The first two rows are for PMM measurements of a red plate transformed into DSS measurements of the same red plate. The bottom two rows are for PMM measurements of a blue plate transformed into DSS measurements of a red plate. The left panels show the residuals before a two-dimensional correction was applied, and the right panels after the correction was applied (see text).

Morrison et al. 2001 and references therein). These are particularly large and difficult to model when an entire plate of $6.5 \times 6.5$ is being mapped into astrometric catalogs. For our purposes, however, we are using only small regions on a plate, which can be mapped into one another and into an external system, such as the du Pont plates, for example, by using up to third-order polynomials. As for magnitude-dependent systematics (i.e., the magnitude equation), these are minimized by using stars within a relatively narrow magnitude range, toward the faint end of the plate limit: $V \sim 15-19$.

The old 60 inch plates were not precorrected in any manner, as they are the least well understood. We are aware that coma is present from the shape of the images on the plates and that it affects the astrometry from coordinate transformations of long into short exposures. In $\S 2.6$ we describe the way in which these plates were incorporated into the proper-motion determinations.

\subsection{Proper-Motion Determinations}

The lists of coordinates from the 60 inch, POSS-I, and $4 \mathrm{~m}$ plates are matched with the list from the du Pont plates. The matching radius is $2^{\prime \prime}$. This basically limits our catalog to objects with proper motions smaller than $\sim 50$ mas $\mathrm{yr}^{-1}$. Although stars with proper motions larger than this value can be recovered from the current lists of positions for all measured objects on each plate, it is not our immediate goal to do so.

The proper motions are determined differentially, by adopting one plate as the master plate, into which all the others are mapped. The master plate is chosen from the du Pont plates, which are best understood and modeled. During long exposures, guiding-induced magnitude-dependent systematics (also known as the magnitude equation; see, e.g., Majewski 1992; Guo et al. 1993; Girard et al. 1998) affect practically all photographic material. However, the du Pont plates have a short exposure, and we assume that this exposure is not strongly affected by the magnitude equation. Therefore, we transform the long exposures into one short exposure, and the magnitude-dependent trend of the residuals is used to correct the long-exposure measurements. This essentially corrects the bright stars $(V \leq 16)$, over which the trend is apparent. One of the magnitude-equation-corrected $\mathrm{du}$ Pont long exposures is then chosen as the master plate. The remaining plates are then transformed into the master plate using up to fourth-order polynomial coordinate transformations and linear color terms. These high-order geometric terms are present most likely due to our inability to accurately determine the center of distortion (see $\S 2.5$ ).

Preliminary proper motions are calculated based on the du Pont and POSS-I plate measures, and, for a handful of fields, from du Pont and $4 \mathrm{~m}$ plates. The transformation of POSS-I plates into the du Pont system occasionally requires third-order polynomials in the coordinates and linear color terms. The derived preliminary proper motions allow propagation back in time to the epoch of the 60 inch plates, and thus, the 60 inch plates are tied to the system of the du Pont master plate. These transformations include third-order polynomials in the coordinates, coma terms, and linear color terms. New proper motions are then calculated from the entire set of plates, and one more iteration is performed to obtain the final values. The reference system that is used to determine the plate transformations consists of faint stars, varying in number from a couple hundred to a couple thousand, depending on the Galactic latitude of the field and the depth of the corresponding plates. The resulting proper motions are relative; the correction from relative to absolute proper motions is derived from the offset defined by the mean proper motion of QSOs and galaxies in the field. We note that the du Pont and 60 inch short 
exposures provide measurements of stars as bright as some of the faintest Hipparcos stars $(V \sim 8)$. However, we do not rely on any of the stars at the bright end to determine the correction to absolute proper motions, because we believe our proper motions at the bright end $(V<15)$ are affected by unaccounted-for magnitude-dependent biases. The proper motions of galaxies and the QSOs are also used at each iteration to check magnitudeand color-dependent systematics. In some of the areas that have $4 \mathrm{~m}$ plates, galaxies are used to correct small trends with color and magnitude left in the first-iteration set of proper motions. QSOs and galaxies are selected from the SDSS (Schneider et al. 2005; DR4 classification). According to the SDSS documentation, the galaxy classification is quite reliable down to $r=21$. For areas that do not overlap with SDSS we select galaxies from a visual inspection of the deepest du Pont plate.

We calculate a proper motion for each object that has at least three measurements separated in time by at least $\sim 40 \mathrm{yr}(\sim 20 \mathrm{yr}$ for fields that include $4 \mathrm{~m}$ plates). The proper motion is calculated for each object from a linear least-squares fit of positions as a function of plate epoch. The formal proper-motion uncertainty is given by the scatter about this best-fit line. Measurements that differ by more than 0.2 from the best-fit line are excluded. Objects that have only three measurements should, however, be considered with caution because they may have unrealistically small formal uncertainties.

\subsection{Proper-Motion Uncertainties}

There are two ways to estimate externally the proper-motion uncertainties: (1) by direct comparison with another high-quality proper-motion catalog and (2) from the proper-motion scatter of objects that have no or negligible intrinsic proper-motion dispersion. For the first test we compare our proper motions with those in the Munn et al. (2004) catalog in five SA fields. We remind the reader that the Munn et al. (2004) proper-motion catalog was made by combining USNO-B (Monet et al. 2003) with the SDSS (DR1). Munn et al. (2004) have used SDSS galaxies to correct for position-dependent proper-motion systematics and to place the proper motions in an absolute reference frame. In Figure 4 we show proper-motion differences (i.e., our relative proper motions minus the proper motions from Munn et al. [2004]) as a function of magnitude. Units for proper motions are mas $\mathrm{yr}^{-1}$ throughout the paper. The left panels show proper-motion differences along right ascension, and the right panel along declination. It is apparent that, in all fields, there is a larger scatter in the right ascension proper-motion differences than in the declination. Proper-motion differences plotted as a function of positions and colors show that the larger scatter in right ascension is not due to systematics related to these quantities. Our proper motions for galaxies and QSOs (i.e., objects with no intrinsic proper-motion dispersion) do not indicate that our measurements are consistently poorer in the right ascension direction than in declination (see below). Interestingly, the histogram of QSO proper motions in Munn et al. (2004, their Figs. 1 and 3) shows that the right ascension proper-motion dispersion is larger than that in declination, in agreement with our findings. From coordinate transformations of the modern du Pont plates directly into SDSS positions in some 13 SAs, we do not find indications that the positional error in right ascension is larger than that in declination. This leads one to conclude that the positional precision in the USNO-B catalog is poorer in right ascension than in declination. Indeed, Figure 1 in Munn et al. (2004), which shows the distribution of QSOs' proper motions in right ascension and declination for both the USNO-B catalog and the new Munn et al. (2004) catalog, indicates that the scatter in right ascension is larger than that in declination in both catalogs.

From our comparison with the Munn et al. (2004) catalog, we obtain a scatter of $\sim 3.8$ mas $\mathrm{yr}^{-1}$ in right ascension and

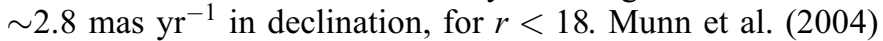

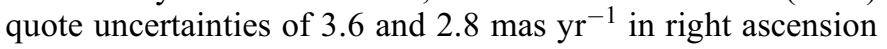
and declination, respectively, for the same magnitude range. This indicates that our uncertainties are substantially smaller. The fact that we see in the proper-motion differences (Fig. 4) the larger scatter in right ascension than in declination, which is characteristic of the Munn et al. (2004) catalog, implies that the errors in the latter catalog dominate the scatter in the proper-motion differences.

Our second test uses galaxies, QSOs, and stars in known streams or clusters, i.e., objects for which the proper-motion dispersion reflects only the measurement uncertainty. In practice, galaxies have larger proper-motion uncertainties than stars due to poor centering of their fuzzy, low-gradient image profiles, and there are very few QSOs in each SA field. Thus, these uncertainty estimates should also be viewed as conservative numbers. In Figure 5 we show relative proper motions of galaxies (open circles) and QSOs (filled red circles) as a function of magnitude for the same SA fields as shown in Figure 4 (the left panel is for right ascension, and the right panel is for declination). Clearly, SA 94 is better measured, and this is because it includes $4 \mathrm{~m}$ plates.

Figure 5 indicates that the proper-motion uncertainty varies considerably with magnitude, and there may be slight variations from field to field. These variations are primarily due to the image quality of the old POSS-I plates. For a typical field we obtain

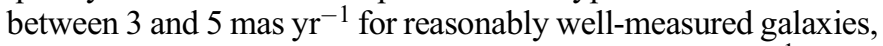
while for fields that have $4 \mathrm{~m}$ plates, we obtain $2-3{\text { mas } \mathrm{yr}^{-1}}^{-1}$. Most fields have on the order of 100 galaxies; therefore, the uncertainty in the correction to absolute proper motion is $\leq 0.5$ mas yr$^{-1}$. For the QSOs it is more difficult to reliably estimate uncertainties due to small-number statistics and the fact that most QSOs are toward the faint limit of the survey. However, for SA 94 we

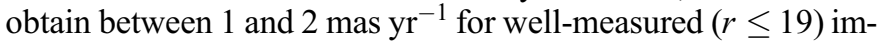
ages. Proper-motion uncertainty estimates as derived from "known" tidal structures are given in $\S 2.8$, in which we present the tidal tail results.

Finally, in Figure 6 we show the correction to absolute proper motion as given by a number of calibrating objects for SA 101 . Most of the fields show similar characteristics. Toward the faint end, the galaxies (green symbols) and QSOs (red symbols) dominate. At the bright end we show the correction to absolute proper motion as given by proper-motion differences of Tycho-2 stars (Høg et al. 2000; filled triangles), UCAC2 stars (open circles), and NPM1 stars (Lick Northern Proper-Motion Program; Klemola et al. 1987; filled circles). For all three of these catalogs, the stars matched with our survey are at the faint limit of the catalog. Thus, these stars have the largest measurement errors as given by any of the three catalogs. Indeed, the proper-motion scatter is between 4 and 6 mas $\mathrm{yr}^{-1}$, poorer than that of our measured galaxies. This is one reason why we have not used Tycho-2, UCAC2, and NPM1 stars to calibrate to absolute proper motions. The second reason is due to obvious offsets between the zero point as determined by galaxies and QSOs in our survey, and that determined based on brighter stars in these external catalogs. This is due to residual magnitude-dependent systematics in either or both of the listed catalogs and our survey. Since the magnitude range of galaxies and QSOs better matches that of our survey stars, using these to establish the absolute proper-motion correction 

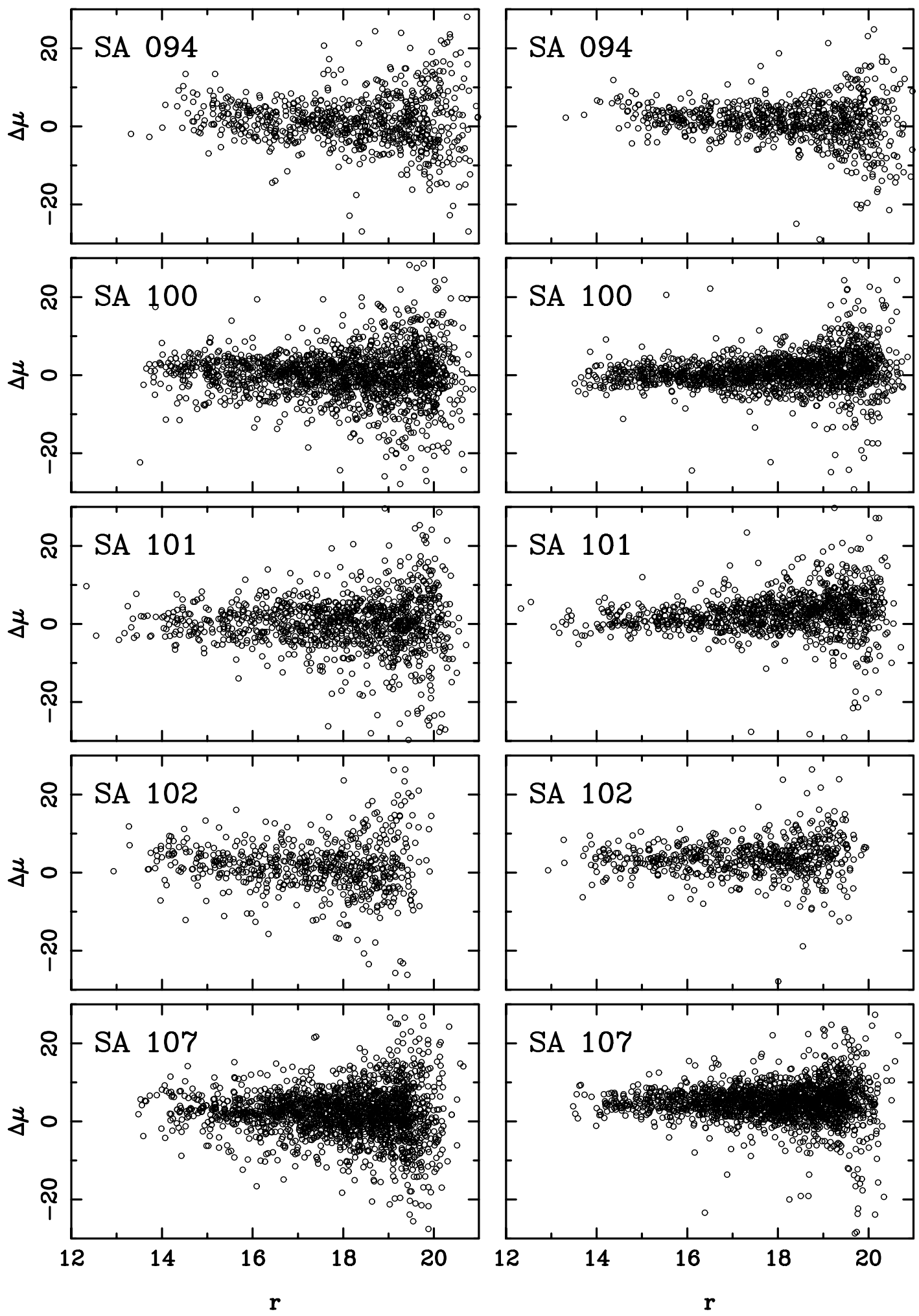

FIG. 4.- Proper-motion differences (ours minus those in the Munn et al. 2004 catalog) as a function of magnitude in five SAs. The left panels show the right ascension direction, and the right panels the declination direction.

minimizes magnitude-dependent systematics. We use both galaxies and QSOs in the determination of the absolute propermotion correction.

One more question remains: Why not use stars in the Munn et al. (2004) catalog as astrometric zero-point calibrators? From comparisons of the zero point as determined by galaxies and by the Munn et al. (2004) stars, we find differences between 0.2 and

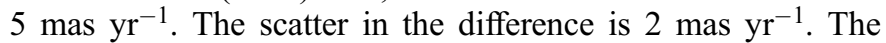
absolute proper-motion calibration in Munn et al. (2004) is also based on SDSS-selected galaxies, and their first-epoch material is in our time series. Nevertheless, differences appear in the two independently determined catalogs. We feel our proper motions 

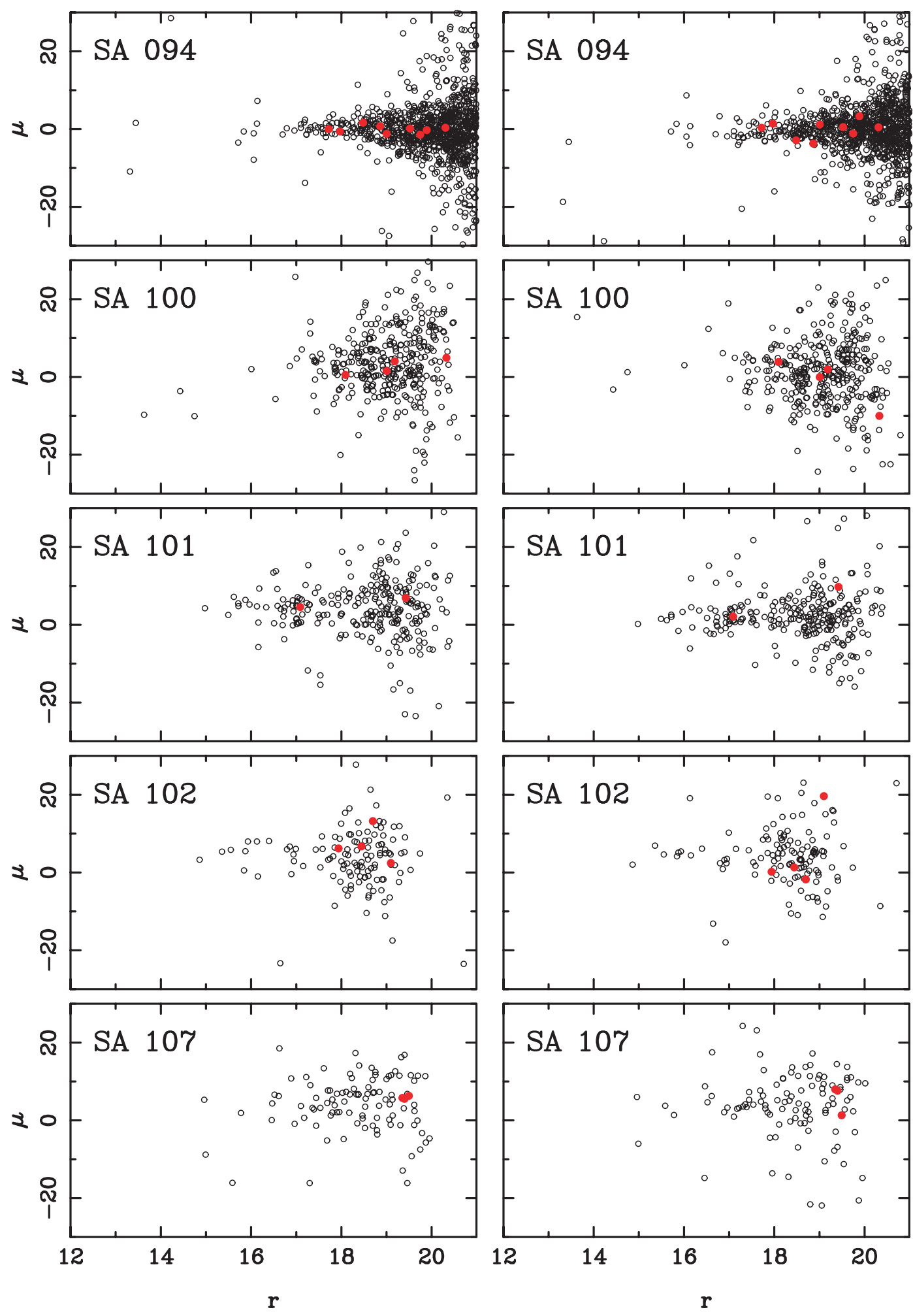

FIG. 5.- Relative proper motions of galaxies (open circles) and QSOs ( filled red circles) as a function of magnitude for five SAs. The left panels show the right ascension direction, and the right panels the declination direction.

are more reliable for two reasons: (1) the modern epoch consists of precise measurements of the high-quality du Pont plates, and (2) the old epoch, i.e., the POSS-I scans, were rereduced here in a very careful manner to minimize position-dependent systematics (see $\S 2.5$ ), while the Munn et al. (2004) proper motions necessarily had to rely on the batch-reduced PMM scans of the POSS-I plates for nearly $10^{9}$ objects. For these reasons, as well as because of the effect seen in Figure 4, we use our internal zeropoint calibration based on galaxies and QSOs.

\subsection{Completeness}

In Figure 7 we show the distribution of objects detected as a function of magnitude in two fields: SA 107, a typical field in our survey, and SA 94, a deep field that includes $4 \mathrm{~m}$ plates. The 


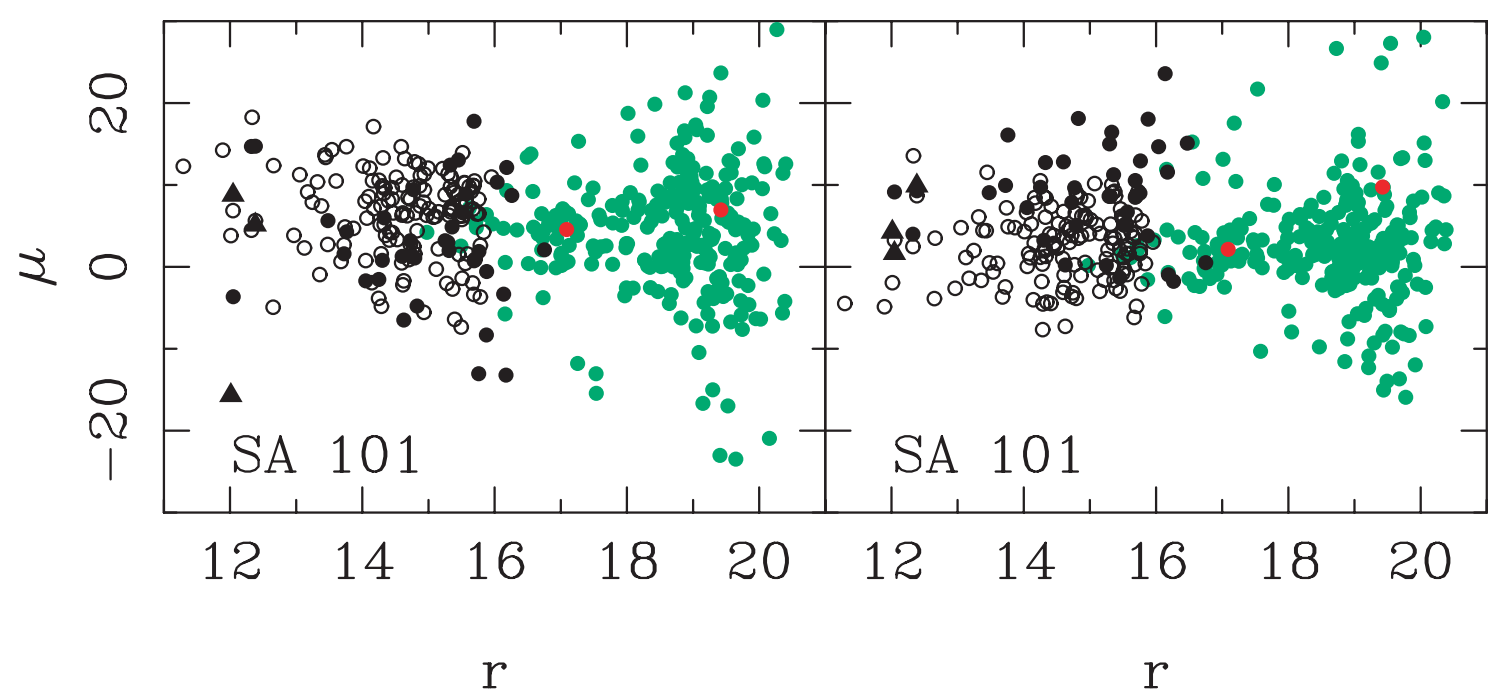

FIG. 6.-Correction to absolute proper motion as a function of magnitude as given by QSOs (red circles), galaxies (green circles), NPM1 stars (black filled circles), UCAC2 stars (open circles), and Tycho-2 stars ( filled triangles). As in the previous two figures, the left panel shows the right ascension direction, and the right panel shows the declination direction.

SDSS distribution is represented with a red line and our survey with a black line, and the hatched area is that fraction of our stars that were measured on the old 60 inch plates. For a typical survey field, our catalog is nearly complete to $r=19$, and this magnitude limit varies slightly from field to field depending on the deepness of the POSS-I plates. Figure 7 shows that the SDSS distribution and our catalog distribution follow each other closely.

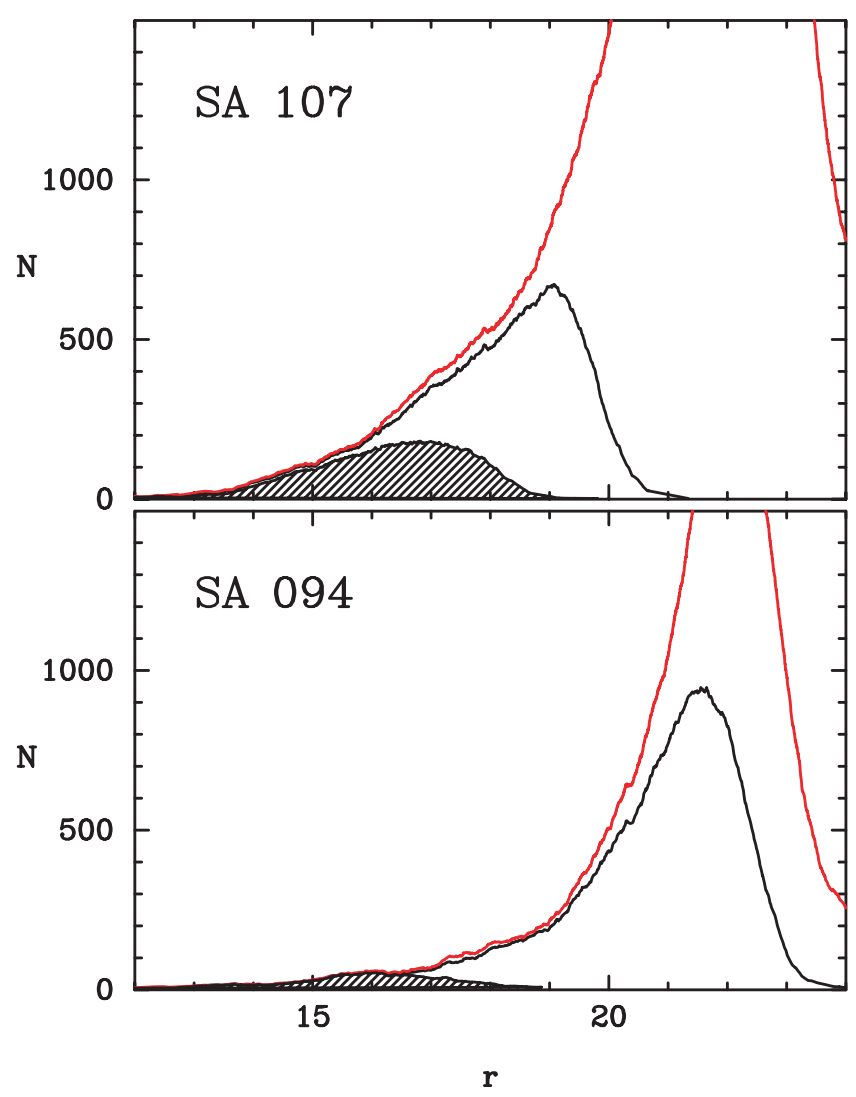

FIG. 7.-Distribution of objects as a function of magnitude for SAs 107 and 94. The SDSS catalog is represented with a red line, and our survey with a black line. The hatched area is our survey, only for objects measured on the old 60 inch plates.
A difference between the SDSS and our catalog counts is, however, present between $r=17$ and 19. This difference indicates that SDSS has $6 \%-9 \%$ more counts than our catalog. For these two fields, we have checked by hand the objects that appear in SDSS and do not appear in our catalog. We found that the absence of these objects from our catalog is due to two reasons: (1) from its construction $(\S 2.6)$ our catalog misses high proper motion (greater than $\sim 50$ mas yr$^{-1}$ ) stars, and (2) SDSS has a nonnegligible number of spurious detections near bright stars that, on inspection of the SDSS images, appear to be located on diffraction spikes. We note that the latter objects have SDSS flags that qualify them as real, primary detections (i.e., flag "GOOD"). To correctly quantify the contribution of each of these effects is not a trivial matter. If we assume, however, that the SDSS represents the true counts, we then obtain a conservative estimate of our completeness limit that is greater than $90 \%$ to $r=19$. The limiting magnitude at $50 \%$ completeness is $r \sim 19.5$. For fields that include $4 \mathrm{~m}$ plates, the catalog is near complete (greater than $90 \%$ ) to $r \sim 21$ and reaches a limiting magnitude of $r=22$ at $50 \%$ completeness.

\section{RESULTS}

In what follows we show proper-motion results in SA fields containing some currently known halo substructures. Quantitative analyses that involve absolute proper motions combined with other information and with models will be presented elsewhere.

\subsection{Sagittarius Tidal Tails}

Based on Hess diagrams, Newberg et al. (2002) have identified overdensities of F-colored stars related to debris from the Sagittarius dwarf in the regions labeled S167-54-21.5 (their Fig. 7), and S341+57-22.5 (their Fig. 5). The latter region includes our SAs 105 and 106. We find, however, very little evidence of clumpiness in the proper-motion diagram because Sgr's main-sequence turnoff is at very faint magnitudes $(r=22.3)$. A small number of Sgr red clump stars at $r \sim 19.5$ may be present in SAs 105 and 106, but these are at the faint end of our survey and will have large proper-motion uncertainties. This SDSS region samples distant parts of the leading tidal tail of Sgr (see Fig. 1 and, e.g., Majewski et al. 2003). Thus, we no longer consider these fields here. 

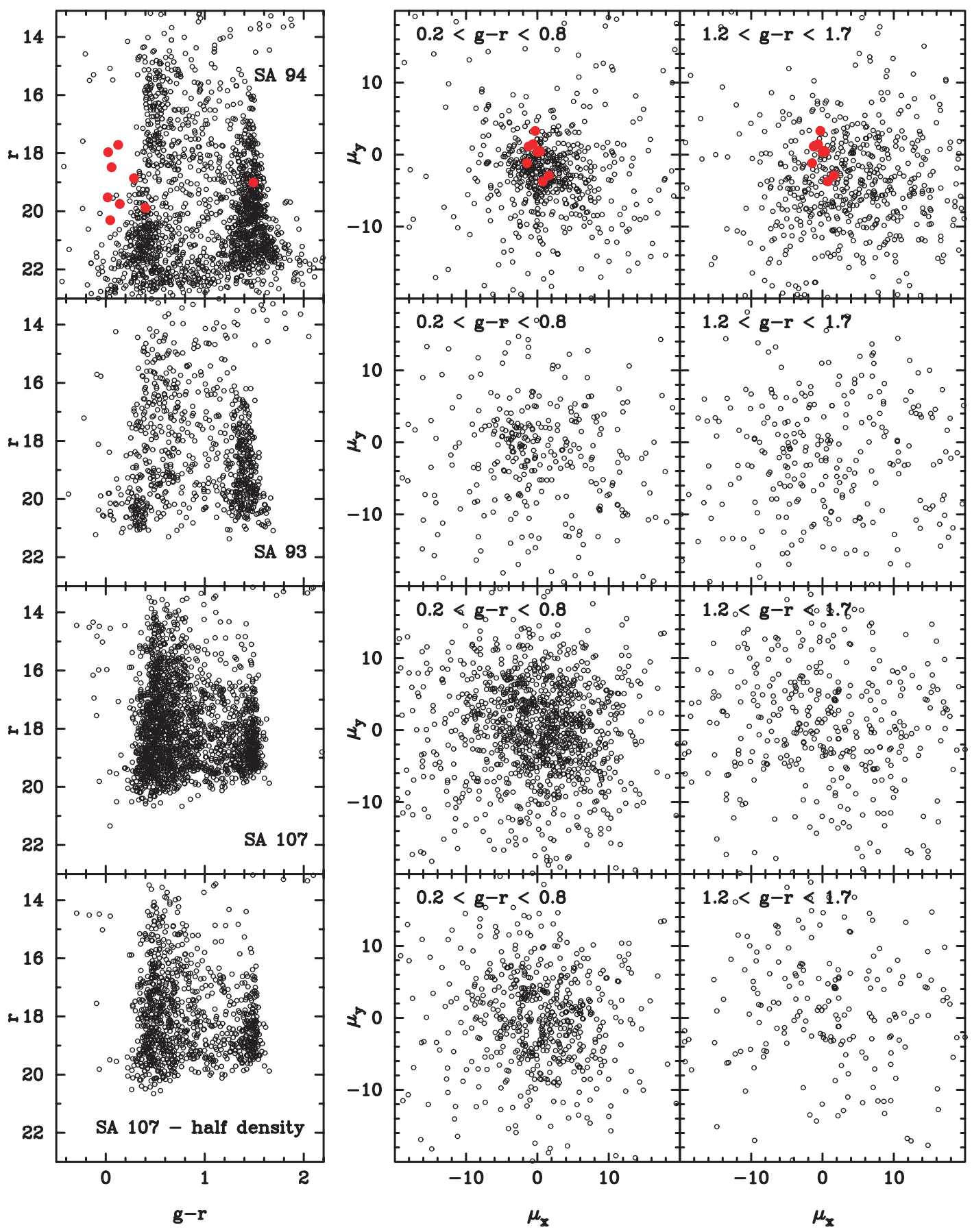

FIG. 8.-CMDs (left panels) and relative proper-motion diagrams (middle and right panels) for stars and QSOs in SAs 94, 93, and 107. The middle and right panels show the proper motions for blue $[0.2<(g-r)<0.8]$ and red $[1.2<(g-r)<1.7]$ stars, respectively. In the top row, QSOs are highlighted with red symbols for comparison with stars' distributions. The bottom row shows SA 107, for which only every other star is plotted to mimic a lower density field.

The SDSS region S167-54-21.5 includes SA 93 at $(l, b)=$ $\left(154^{\circ} .2,-58^{\circ} .1\right)$ and SA 94 at $(l, b)=\left(175^{\circ} .3,-49^{\circ} .2\right)$, and it samples the trailing tidal tail of Sgr, which is generally closer to the Sun than the leading trail (e.g., Majewski et al. 2003). Yanny et al. (2003, 2004), Majewski et al. (2004), and Law et al. (2005) confirm with radial velocities the presence of Sgr debris in this region. In Figure 8 we show the SDSS CMDs and relative propermotion distributions for SAs 94, 93, and 107. The middle and right panels show proper motions: the $x$-direction corresponds to proper motions along right ascension, and the $y$-direction to declination. Each row represents one field. SA 107, located at $(l, b)=\left(5^{\circ} 7,+41^{\circ} .3\right)$, was chosen for comparison with the other two fields, and it is roughly symmetrically placed in the Galaxy with SA 94. The reddening is relatively low in all fields: for SA 93 it is $E(B-V) \sim 0.03$, for SA 94 it is 0.09 , and for SA 107 it is 0.11 (Schlegel et al. 1998). The CMDs show objects in the SDSS that were matched with our proper-motion survey. Galaxies according to the SDSS classification were eliminated. The magnitudes and colors were not dereddened. For SA 94, for which we have better statistics for the QSOs than in the other fields, we highlight the QSOs with red symbols so that their proper-motion distribution can be compared to that of stars.

The middle panels show the proper-motion distribution of blue stars $(0.2<g-r<0.8)$ chosen to represent the turnoff of 
thick-disk and halo stars, and the right panels that of red stars $(1.2<g-r<1.7)$ chosen to represent more nearby, disk dwarf stars. Sgr's turnoff is visible in the CMDs of SAs 94 and 93, at $r \sim 21$. SA 107 is also located in the SDSS region S6+41-20, which Newberg et al. (2002) find is most consistent with a smooth halo/thick-disk population. Because of its direction toward the inner Galaxy and the fact that the scale length of the thick disk is 3-4 kpc (e.g., Juric et al. 2006), SA 107 samples more thick-disk stars than SA 94. The proper-motion distributions show the intrinsic dispersion convolved with the proper-motion measurement uncertainty, which increases with magnitude.

By comparing the Figure 8 proper-motion distribution of presumably nearby red stars in all fields, it is clear that SA 94 is a much better measured field - a result of having $4 \mathrm{~m}$ plates in its time series data. The proper-motion uncertainties for SAs 93 and 107 are, however, comparable to one another. The proper-motion distribution in SA 107 shows a clear kinematical distinction between blue stars, i.e., distant thick-disk and halo stars near the main-sequence turnoff, and red, nearby dwarf stars. The fourth row shows the same diagrams as the third row, but with only half the stars in SA 107 plotted so as to approximately match the number of stars in SA 94 in the appropriate magnitude range. It is evident that the blue stars in SAs 94 and 93 show more concentrated clumping in proper motions due to the presence of a population with a dispersion much tighter than that expected for random thick-disk/halo stars (i.e., SA 107).

This identified proper-motion clumpiness together with a sudden overdensity of stars at a particular magnitude in the CMD is a clear signature of distinct substructure in the halo, presumably dominated by Sgr tidal debris. In SA 94, the best-measured field, the proper-motion scatter of the clumped, blue stars is $\sim 1.8-2.0$ mas $\mathrm{yr}^{-1}$. This number is determined primarily by faint stars $(r=21)$ and reflects our proper-motion uncertainty. In this contribution we present the mean absolute proper motion of candidate Sgr debris in SAs 94 and 93.

In SA 94 the correction to absolute proper motion is given by the error-weighted mean of two determinations: that with respect to QSOs and that with respect to galaxies. The former determination is the average of nine QSOs, $\mu_{\alpha} \cos \delta=-0.08 \pm$ $0.32 \mathrm{mas} \mathrm{yr}^{-1}$ and $\mu_{\delta}=-0.07 \pm 0.73 \mathrm{mas} \mathrm{yr}^{-1}$. The latter includes 885 galaxies with magnitudes between $r=15$ and 21 that have measurements on at least four plates and proper-motion val-

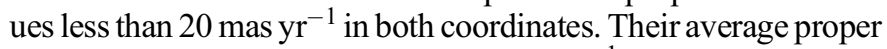

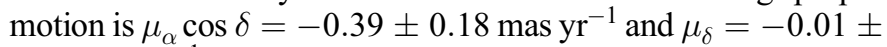
$0.19 \mathrm{mas} \mathrm{yr}^{-1}$. The error-weighted average of these two determinations is $\mu_{\alpha} \cos \delta=-0.32 \pm 0.16 \operatorname{mas~yr}^{-1}$ and $\mu_{\delta}=0.00 \pm$ $0.18 \mathrm{mas} \mathrm{yr}^{-1}$. Candidate Sgr stream stars are selected based on the SDSS CMD (Fig. 8) to belong to the turnoff region. Only stars with measurements on at least four plates and with $r$ magnitudes brighter than 21.7 are included, to avoid stars with highly uncertain proper motions. Furthermore, stars with proper-motion

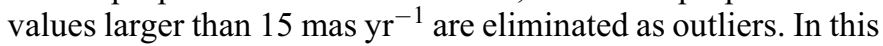
way, the sample of Sgr candidates consists of 156 objects. To determine their mean and dispersion, we have used the probabilityplot method (Hamaker 1978) using the inner 80\% of the propermotion distribution. By doing so we aim to eliminate the minor contribution of distant halo stars to the estimate of the mean proper motion of the candidates. The Besancon model (Robin et al. 2003) predicts 21 halo stars in the CMD region and propermotion range used to select Sgr debris candidates. Therefore, the chosen contamination fraction of $20 \%$ seems to slightly overestimate the Besancon model output. We find the mean relative proper motion of Sgr debris candidates in SA 94 to be $\mu_{\alpha} \cos \delta=$

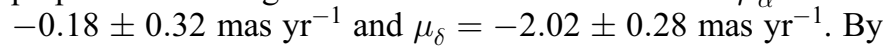

using fractions between $60 \%$ and $90 \%$, the estimate of the mean does not change within its formal uncertainty. The mean absolute proper motion for candidate Sgr debris in SA 94 is

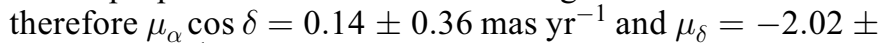

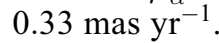

For SA 93 we also present a preliminary value for Sgr stream candidates. We plan to improve the determinations for both SA 93 and 94 by obtaining radial velocity data that will help establish membership in the stream. For SA 93 the correction to absolute proper motion is given by galaxies and QSOs together, rather than two independent measurements, as was done for SA 94. This is because there are only two QSOs measured in this field, one of which is a galaxy as well. Proceeding in a similar manner as for SA 94, we obtain the correction to the absolute proper motion as given by the mean of 222 extragalactic objects: $\mu_{\alpha} \cos \delta=$

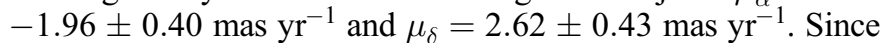
there are far fewer Sgr candidates in SA 93 than in SA 94, we have made use of all stars within $r=14-20$ and $(g-r)=0.0-0.9$ to determine the mean motion, since some subgiant and red clump stars may also be present besides the turnoff stars. In this case the halo contamination is much more important than in SA 94, and probability plots will not give an accurate result. We have thus simply selected the candidates by defining a very conservative

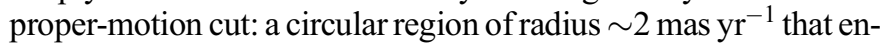
compasses the proper-motion clump seen in Figure 8. The mean is thus based on 63 stars, and it is $\mu_{\alpha} \cos \delta=-2.54 \pm 0.18$ mas yr$^{-1}$ and $\mu_{\delta}=0.30 \pm 0.24$ mas $\mathrm{yr}^{-1}$. The uncertainties are derived from the scatter given by the $63 \mathrm{Sgr}$ candidates. We caution that this uncertainty is formally low because it does not account for the uncertainty in choosing Sgr stream members. Finally, the absolute proper motion for Sgr candidates in SA 93 is $\mu_{\alpha} \cos \delta=$

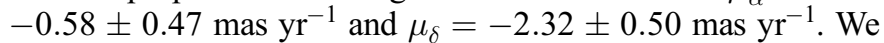
regard this number as preliminary, and with uncertainties possibly underestimated. In both SA 94 and 93 determinations the membership in the stream is the major source of error.

Majewski et al. (2006) have shown that by measuring the proper motion of Sgr debris, especially along the trailing tail, one can determine the rotation velocity of the local standard of rest (LSR), whereas Law et al. (2005) and Johnston et al. (2005) show how the dynamics of the Sgr arms are also affected by the flattening, $q$, of the Galactic potential. In Figures 9 and 10 we show proper-motion predictions by Law et al. (2005) for Sgr tidal debris in the direction of SAs 93 and 94 compared to the observed results found here in these two fields. The proper motions are shown as a function of the longitude along Sgr's orbit (Majewski et al. 2003); $\Lambda=0^{\circ}$ corresponds to the main body of Sgr, and $\Lambda$ increases in the trailing direction. In Figure 9 the adopted velocity of the LSR is $220 \mathrm{~km} \mathrm{~s}^{-1}$, while the flattening of the halo $q$ has three values, corresponding to prolate (top), spherical (middle), and oblate (bottom) halos. In Figure 10 the adopted flattening of the halo is $q=0.9$, and the velocity of the LSR varies from 260 to $180 \mathrm{~km} \mathrm{~s}^{-1}$, as indicated in each panel. The general agreement between our proper-motion results and the model, which was constrained solely from the distribution and radial velocities of $M$ giants (Majewski et al. 2003, 2004) and a given Galactic potential (i.e., no proper-motion data), is remarkable. This correspondence reinforces the assumption that we are measuring proper motions in Sgr's tidal stream. We note that the proper-motion range shown in Figures 9 and 10 is only $\sim 3$ mas $\mathrm{yr}^{-1}$, a typical value for the proper-motion uncertainty per well-measured star in catalogs such as Tycho-2 (Høg et al. 2000) and the Third Installment of the Southern Proper Motion Program (Girard et al. 2004). To better illustrate this, we include here the mean absolute proper motion of red field stars (see also 

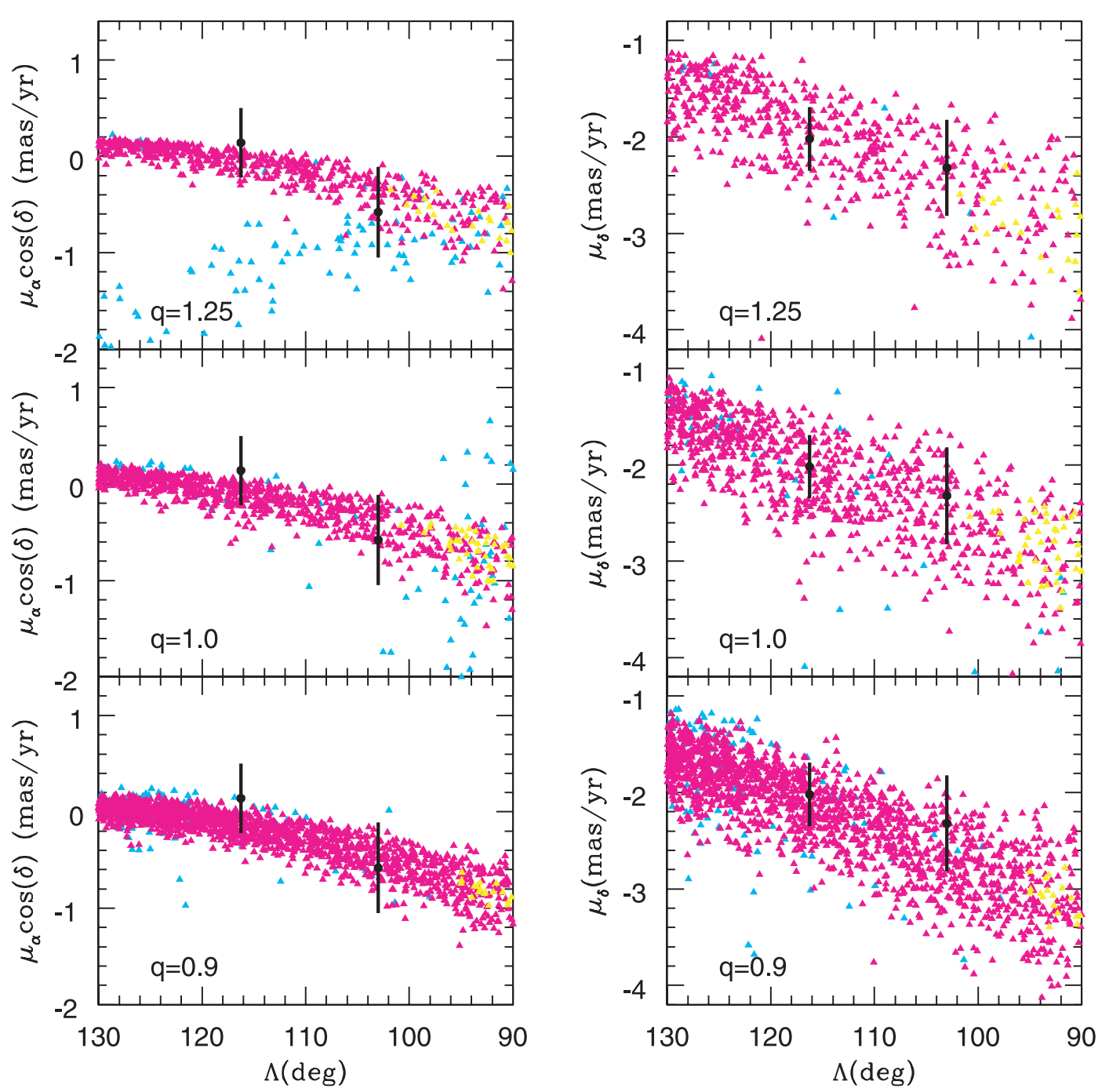

FIG. 9.- Proper motions along Sgr's southern trailing tail as predicted by the Law et al. (2005) models (colored triangles). The dark symbols with $1 \sigma$ error bars show our preliminary results in SA $93\left(\Lambda=103^{\circ}\right)$ and SA $94\left(\Lambda=116^{\circ}\right)$. The LSR velocity adopted for this model is $220 \mathrm{~km} \mathrm{~s}^{-1}$, while the flattening of the halo $q$ varies as specified in each panel. The colored triangles represent $N$-body model particles stripped from Sgr since its last apogalacticon, i.e., the present orbit ( yellow triangles), during the previous orbit (magenta triangles), and two orbits ago (cyan triangles); this color scheme matches that used in Law et al. (2005).

Fig. 8). In SA 94 we obtain $\mu_{\alpha} \cos \delta=2.80 \pm 0.57$ mas $\mathrm{yr}^{-1}$

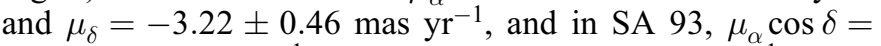
$3.99 \pm 0.88 \mathrm{mas} \mathrm{yr}^{-1}$ and $\mu_{\delta}=-0.94 \pm 0.80 \mathrm{mas} \mathrm{yr}^{-1}$. Thus, along right ascension the mean proper motions of red field stars in both areas lie outside the proper-motion range shown in Figures 9 and 10.

While our preliminary proper motions in only two SA fields do not yet lend discriminatory power between the Galactic models shown, with improved proper-motion samples and the inclusion of additional fields at other $\Lambda$ (e.g., SAs 116 and 117; see Fig. 1) we expect to be able to more rigorously address this issue in the near future.

\subsection{The Monoceros Structure}

In Figure 11 we show plots similar to those in Figure 8 for the fields SA 96 at $(l, b)=\left(198^{\circ} .3,-26^{\circ} .0\right)$, SA 100 at $(l, b)=$ $\left(227^{\circ} .6,+26^{\circ} .7\right)$, and SA 101 at $(l, b)=\left(239^{\circ} .0,+39^{\circ} .9\right)$. These areas sample regions in the Mon structure at the anticenter and across the Galactic plane. For SA 96 the data for the CMD are not in the available SDSS data releases. We obtained the data set from B. Yanny, and the photometry is dereddened for this field. Reddening is, however, rather low for all three of these fields:
$E(B-V)=0.07$ for SA 96, $E(B-V)=0.04$ for SA 100 , and $E(B-V)=0.04$ for SA 101. Since the data for SA 96 are dereddened, we have shifted blueward the color ranges for selecting blue and red stars by $0.1 \mathrm{mag}$ compared to the other fields (Fig. 11). Newberg et al. (2002) designate the region containing SA 96 as S200-24-19.8, for which their Fig. 15 shows a clear main-sequence turnoff at $g=19.8$. SA 100 is located only $3^{\circ}$ away from the eastern edge of S223+20-19.4, which also displays a clear main sequence with the turnoff at $g \sim$ 19.4 (see Fig. 12 in Newberg et al. 2002). At $b \sim 40^{\circ}$, SA 101 is farther away from the Monoceros Ring (see also Fig. 1). The majority of the stars measured in our survey are, however, brighter than these turnoffs and the corresponding mainsequence stars that were studied by SDSS and assigned to the Mon structure.

Yanny et al. $(2003,2004)$ measured radial velocities of candidate turnoff stars in the Monoceros Ring to demonstrate that their dispersion is indicative of a kinematically cold stream (but cf. Crane et al. 2003). In Figure 11 (top panels) the red symbols show the stars in our SA 96 field that have radial velocities measured by Yanny et al. $(2003,2004)$. These stars are at the faint limit of our survey; therefore, their proper motions are quite 

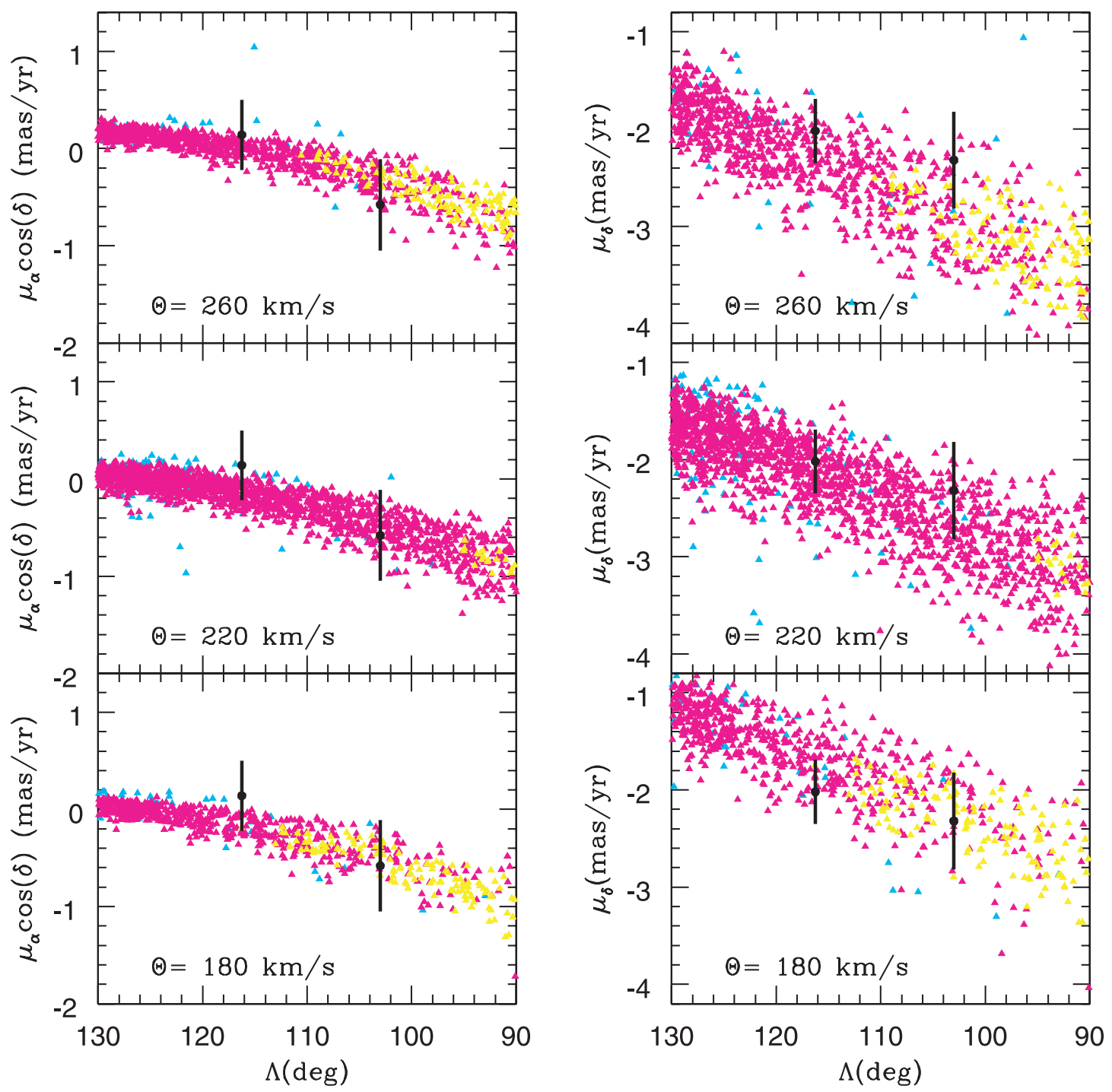

FIG. 10.- Similar to Fig. 9. The flattening of the halo adopted for this model is 0.9 , while the velocity of the LSR varies as specified in each panel. The color representation is as in Fig. 9 and Law et al. (2005).

uncertain. Since the mean radial velocity of the candidate Mon stars overlaps with that of the thick disk (Yanny et al. 2003, 2004), it is also possible that some of the stars are indeed thickdisk stars, i.e., have a larger proper-motion dispersion than that of a cold stream. The tight clump seen in the proper-motion diagram of blue stars in SA 96 is comprised of stars with $r=$ 15-19. Are these only thick-disk/halo stars? Comparing SA 96 with SA 100, which is located at the same latitude as SA 96, only above the plane, and only $\sim 30^{\circ}$ away in longitude from SA 96 , we sample very similar parts of the major Galactic components. However, there is a clear difference in the number of blue stars in the two regions. A careful inspection of Figure 15 in Newberg et al. (2002), which corresponds to SA 96, suggests that multiple main sequences and turnoffs may be present, with the faintest one in SDSS being the most distinct. To better illustrate the proper-motion clumpiness in SA 96, we show a zoomed-in proper-motion diagram of the blue stars (as defined in Fig. 11) for SA 96 (Fig. 12, left panels) and SA 100 (Fig. 12, right panels). The middle and bottom panels show the proper motions as a function of magnitude. The stellar excess in SA 96, as well as the proper-motion tightness, is obvious when compared to SA 100.
The stellar excess is also apparent when comparing the propermotion distributions in the three fields with those predicted by the Besancon Galactic model (Robin et al. 2003), which obviously contains only the major Galactic components. In Figure 13 we show the absolute proper-motion distribution along one coordinate (right ascension, for example) in all three fields as determined from the Besancon Galactic model (Robin et al. 2003) (Fig. 13, top panels) and from our data (Fig. 13, bottom panels). The filter system of the Besancon models is the Canada-FranceHawaii Telescope MEGACAM system, which is very close to the SDSS system. At any rate, we are interested only in relative comparisons between fields, rather than a direct comparison between data and model counts. The model proper motions were convolved with a 1 mas $\mathrm{yr}^{-1}$ proper-motion uncertainty to approximately match the errors of the observed proper motions.

The distributions were constructed for blue and red stars as defined above (see also Fig. 11) and in the magnitude range $r=14-19$. SA 96 is represented with a red line, SA 100 with a green line, and SA 101 with a blue line. Rather than comparing directly the distributions given by the data with those given by the model for each field, we proceed to do a relative comparison as follows: We compare pairs of fields, i.e., take ratios of the 

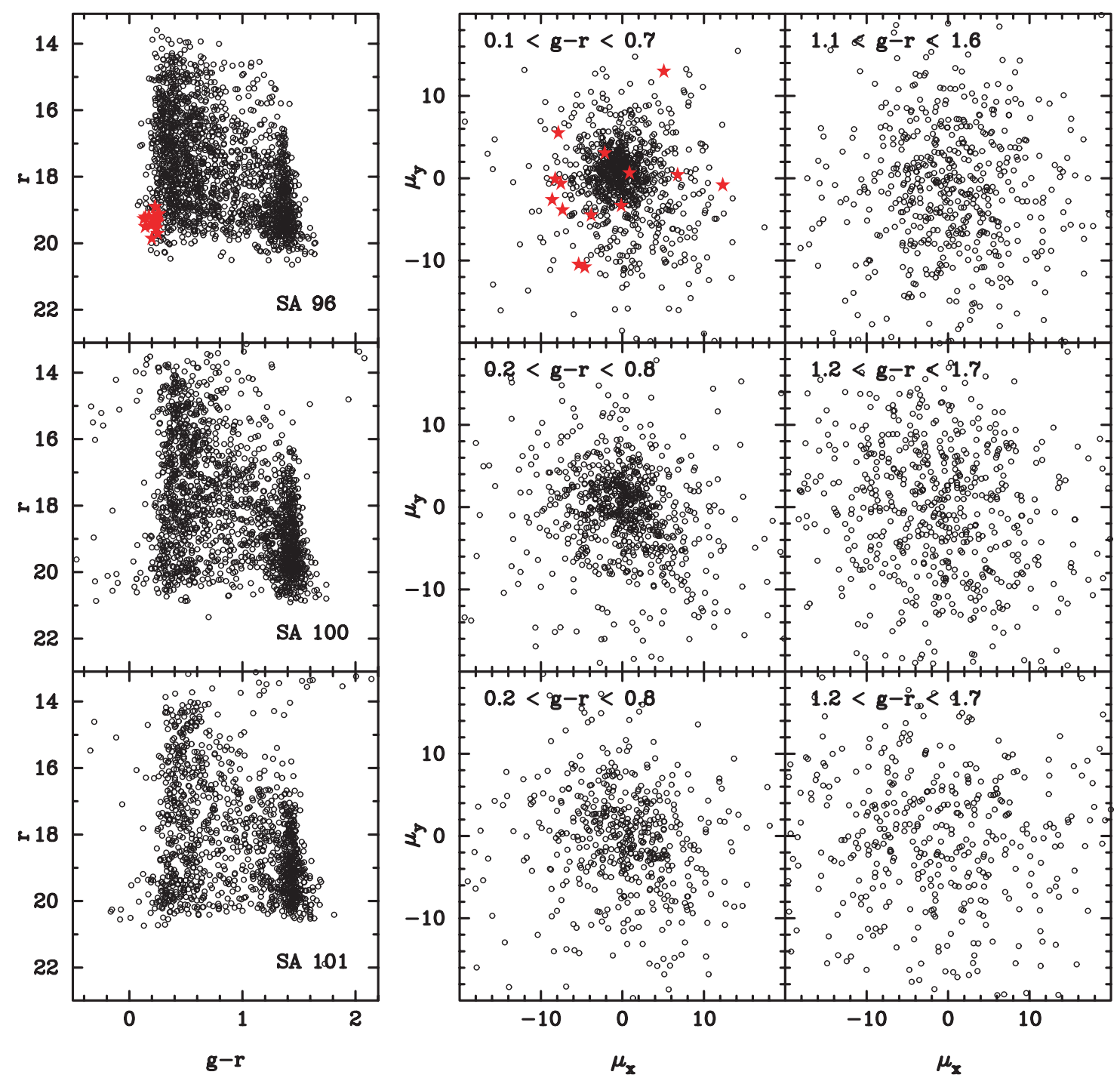

Fig. 11. - Same as Fig. 8, but for SAs 96, 100, and 101. The red symbols in SA 96 show stars with radial velocities measured by Yanny et al. (2003, 2004) in the turnoff of the Mon structure.

distributions as given separately by the data and the model for SAs 100 and 101, and for SAs 96 and 100. These ratios are readily apparent from the plot, as given by the areas under each curve. Alternatively, one can use the ratios of the peaks for pairs of fields. By doing so, for the red stars it is apparent that the observations are in good agreement with the model predictions. For the blue stars, the ratios of the proper-motion distributions for SAs 100 and 101 as given by the model, and the observations are in reasonably good agreement. However, this is not the case for the ratios for SAs 96 and 100. Clearly, the data show that SA 96 has an excess of stars when compared to the model predictions. We also note here the good agreement between model and data for the displacement in absolute proper motion between fields as the Galactic plane is crossed.

One interpretation for the excess counts in SA 96 is that in this region we see multiple, wrapped streams from the Mon structure, with the most distant one detected by SDSS at $\sim 13 \mathrm{kpc}$. Our preliminary absolute proper motion for candidate Mon stars in SA 96 indicates a thick-disk-like motion in agreement with the recently modeled orbit of the Mon system by Peñarrubia et al. (2005). Another possible interpretation is that the excess stars in SA 96 are part of the CMa overdensity, under the assumption that this overdensity is the core of a disrupted dwarf galaxy. The nature of this overdensity is strongly debated in the current literature. Other interpretations besides the dwarf galaxy hypothesis are that the overdensity is the warp of the Galactic disk (e.g., Momany et al. 2006), a spiral arm in the warped disk (e.g., Carraro et al. 2005), or the periphery of a system centered in the region of the Argo constellation (Rocha-Pinto et al. 2006). Currently, no interpretation of the reported CMa overdensity is clearly proved or widely accepted. If CMa proves to be any of the above interpretations other than the dwarf galaxy one, then the excess in SA 96 is very unlikely to be related to the CMa overdensity, primarily because of its location. We therefore explore the possible connection between SA 96 and the CMa overdensity interpreted as a dwarf galaxy.

In a recent wide-area study of the distribution of red-clump stars from 2MASS, Bellazzini et al. (2006) mapped out the overdensity in CMa, as well as the Galactic warp. From their study, CMa stands out as a distinct overdensity in the outer regions of the Galactic disk. More importantly, because it is so nearby, CMa covers a large portion of the sky, and the geometry is such that at lower longitudes, toward the anticenter, $\mathrm{CMa}$ is closer to the $\operatorname{Sun}\left(d_{\odot} \sim 6 \mathrm{kpc}\right)$ than its core, which is supposedly 

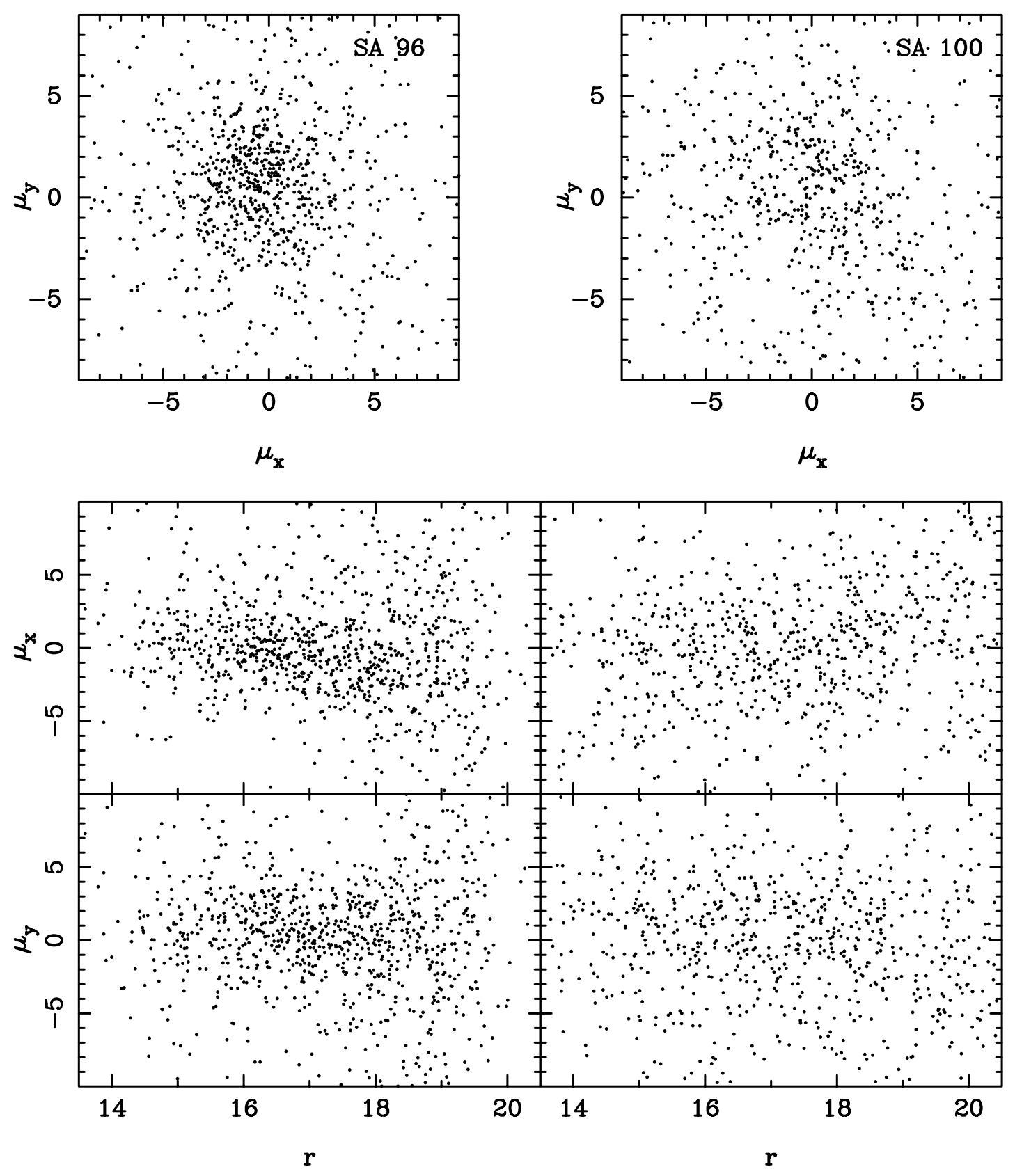

FIG. 12.- Relative proper-motion distributions and proper motions as a function of magnitude for blue stars in SA 96 (left panels) and SA 100 (right panels).

at $(l, b)=\left(240^{\circ},-8^{\circ}\right), d_{\odot} \sim 8 \mathrm{kpc}$ (Bellazzini et al. 2006; Martínez-Delgado et al. 2005). Two figures in Bellazzini et al. (2006) support the notion that SA 96 may be on the outskirts of the reported CMa overdensity: (1) their Figure 9 (top), which shows the excess of the surface density of CMa and indicates that CMa is extended away from the Galactic plane at longitudes closer to the Galactic anticenter; and (2) Figure 13 (bottom), which shows a Sgr-type galaxy placed at the distance and location of CMa. Indeed, its putative extension is impressive, and SA 96 would lie at the tidal radius of such a system. Another indication that the system may be elongated in the direction of Galactic rotation and away from the plane is the motion of CMa as measured by Dinescu et al. (2005b): the $\Theta$-velocity component is $188 \pm 15 \mathrm{~km} \mathrm{~s}^{-1}$, while the $W$-component is $-49 \pm 15 \mathrm{~km} \mathrm{~s}^{-1}$. Radial velocities of our proper-motion stars in the field of SA 96 should help clarify this issue and hopefully lend further clues to the nature of the Mon and CMa structures.
We proceed now to estimate the proper-motion uncertainty by assuming that the blue stars in the proper-motion clump seen in SA 96 belong to a kinematically cold system, and therefore that their proper-motion dispersion reflects measurement errors only. In the proper-motion diagram of SA 96, in Figure 12 there appear to be two overlapping proper-motion clumps with different dispersion: one less tightly clumped, centered at $\left(\mu_{x}, \mu_{y}\right) \sim$

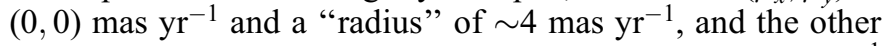
more tightly clumped, centered at $\left(\mu_{x}, \mu_{y}\right) \sim(-1,+2)$ mas $\mathrm{yr}^{-1}$

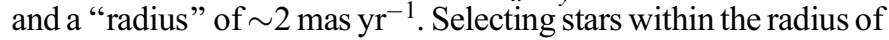
the less tight clump, and within $r=14-18$, we obtain a proper-

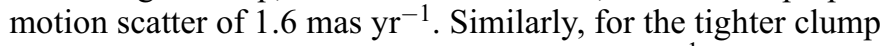
we obtain a proper-motion scatter of $\sim 1$ mas $\mathrm{yr}^{-1}$. These numbers are only approximate estimates to illustrate the precision of the proper motions in this work. A rigorous kinematical understanding and therefore a better separation of these two clumps is beyond the scope of this paper. This will be addressed in future 


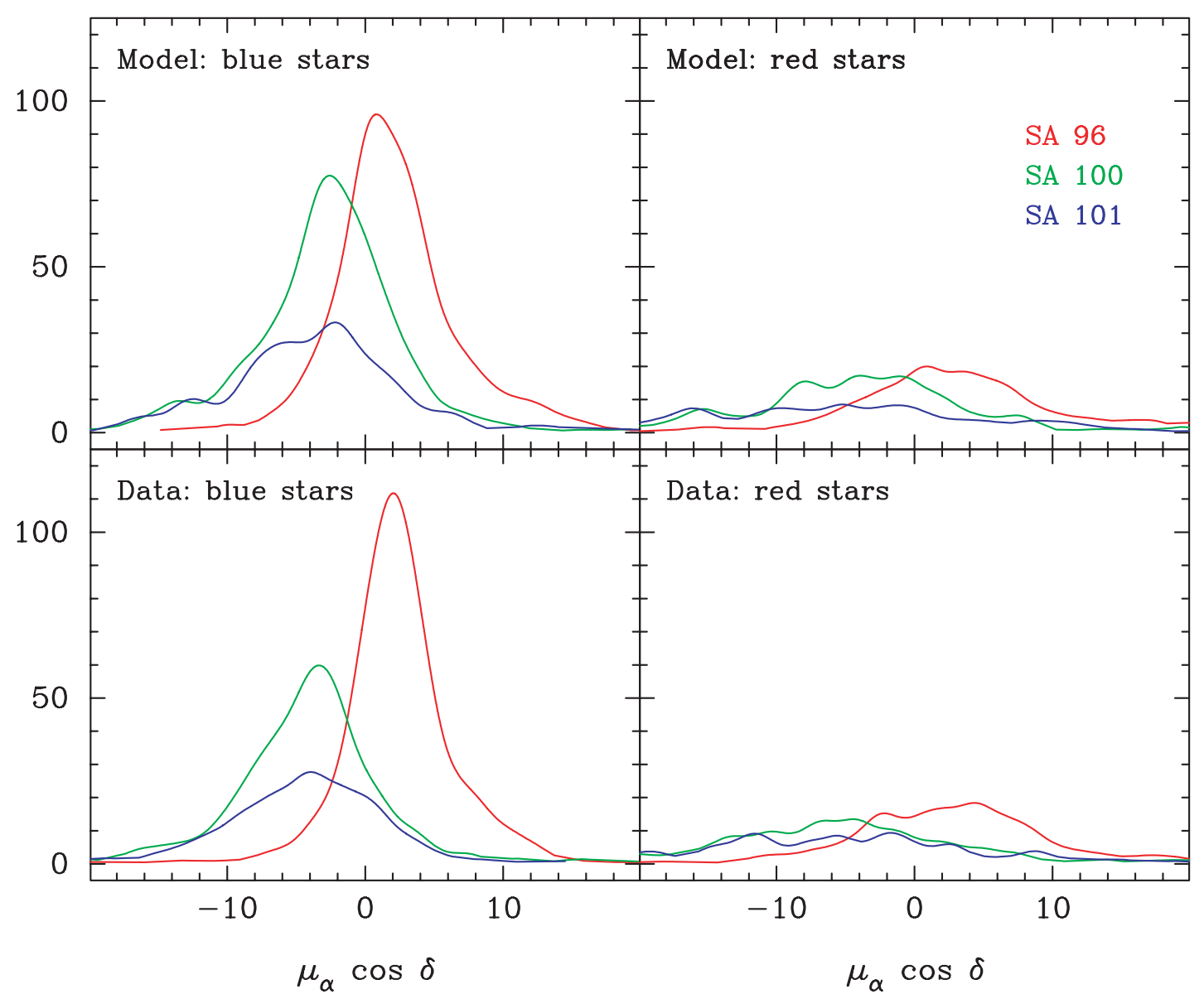

Fig. 13.- Absolute proper-motion distribution along right ascension as given by the Besancon Galactic model (top panels) and by our data (bottom panels). The blue and red stars as selected in Fig. 11 are shown here in the left and right panels, respectively. The magnitude range of the selected stars is $r=14-19$.

work in which radial velocities will also be considered. One more cautionary note should be made: the population of the less tight proper-motion clump seems to appear in SA 100 as well (Fig. 12), and may therefore be representative of distant halo/ thick-disk stars, i.e., a population with a nonnegligible intrinsic proper-motion dispersion.

\section{SUMMARY}

We describe our ground-based, photographic absolute propermotion survey along three near-equatorial declination zones. Proper motions are derived in $40^{\prime} \times 40^{\prime}$ fields from a collection of $2.5 \mathrm{~m}$ du Pont, $4 \mathrm{~m}$ Mayall, 60 inch Mount Wilson, and POSSI plates. The time baseline varies between 40 and $85 \mathrm{yr}$. We have demonstrated that we obtain proper-motion uncertainties be-

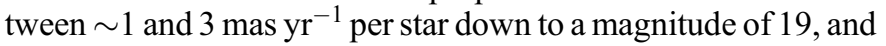
for a few fields down to 21 . The typical uncertainty in the correction to absolute proper motions as given by galaxies and QSOs is between 0.2 and 0.8 mas $\mathrm{yr}^{-1}$. The described proper-motion survey is complemented by our own ongoing radial-velocity and photometric follow-up programs, as well as by current surveys such as SDSS and QUEST. The characteristics of this proper- motion survey make it suitable to address many topics related to both the main Galactic components and the tidal features seen in the halo and associated with streams from disrupted satellites. In this contribution we present preliminary results in the southern trailing tidal tail of Sgr and in the Monoceros Ring region.

We thank Stephen Levine from USNO, Flagstaff, for making available the POSS-I USNO scans. S. R. M. is grateful to the Carnegie Observatories and their former director Augustus Oemler for a Carnegie Visiting Associateship that made possible the collection of the du Pont plates used in this survey. The financial support from NSF grants AST 04-06884 and AST 04-07207 for this research is acknowledged. This publication makes uses of SDSS data products. Funding for the SDSS and SDSS-II has been provided by the Alfred P. Sloan Foundation, the Participating Institutions, the National Science Foundation, the US Department of Energy, the National Aeronautics and Space Administration, the Japanese Monbukagakusho, the Max Planck Society, and the Higher Education Funding Council for England. The SDSS Web site is http://www.sdss.org.
Bellazzini, M., Ibata, R., Martin, N., Lewis, G. F., Conn, B., \& Irwin, M., J. 2006, MNRAS, 366, 865

Bertin, E., \& Arnouts, S. 1996, A\&AS, 117, 393

Carraro, G., Vázquez, R. A., Moitinho, A., \& Baume, G. 2005, ApJ, 630, L153

Chiu, L.-T. 1976, PASP, 88, 803

Chiu, L.-T. G. 1980, ApJS, 44, 31

Cohen, J. G. 2004, AJ, 127, 1545

\section{REFERENCES}

Conn, B. C., Lewis, G. F., Irwin, M. J., Ibata, R. A., Ferguson, A. M. N., Tanvir, N., \& Irwin, J. M. 2005a, MNRAS, 362, 475

Conn, B. C., Martin, N. F., Lewis, G. F., Ibata, R. A., Bellazzini, M., \& Irwin, M. J. 2005b, MNRAS, 364, L13

Crane, J. D., Majewski, S. R., Rocha-Pinto, H. J., Frinchaboy, P. M., Skrutskie, M. F., \& Law, D. R. 2003, ApJ, 594, L119

Cudworth, K. M., \& Rees, R. F. 1991, PASP, 103, 470 
Dinescu, D. I., Girard, T. M., van Altena, W. F., \& López, C. E. 2005a, ApJ, 618, L25

Dinescu, D. I., Majewski, S. R., Girard, T. M., \& Cudworth, K. M. 2000, AJ, 120,1892

Dinescu, D. I., Martínez-Delgado, D., Girard, T. M., Peñarrubia, J., Rix, H.-W., Butler, D., \& van Altena, W. F. 2005b, ApJ, 631, L49

Dinescu, D. I., et al. 2002, ApJ, 575, L67

Duffau, S., Zinn, R., Vivas, A. K., Carraro, G., Méndez, R. A., Winnick, R., \& Gallart, C. 2006, ApJ, 636, L97

Girard, T. M., Dinescu, D. I., van Altena, W. F., Platais, I., Monet, D. G., \& López, C. E. 2004, AJ, 127, 3060

Girard, T. M., Platais, I., Kozhurina-Platais, V., van Altena, W. F., \& López, C. E. 1998, AJ, 115, 855

Guo, X., Girard, T. M., van Altena, W. F., \& López, C. E. 1993, AJ, 105, 2182

Hamaker, H. C. 1978, Appl. Stat., 27, 76

Høg, E., et al. 2000, A\&A, 355, L27

Ibata, R. A., Irwin, M. J., Lewis, G. F., Perguson, A. M. N., \& Tanvir, N. 2003, MNRAS, 340, L21

Ibata, R. A., Irwin, M., Lewis, G. F., \& Stolte, A. 2001, ApJ, 547, L133

Johnston, K. V., Law, D. R., \& Majewski, S. R. 2005, ApJ, 619, 800

Johnston, K. V., Zhao, H., Spergel, D. N., \& Hernquist, L. 1999, ApJ, 512, L109

Jurić, M., et al. 2006, ApJ, in press

Klemola, A., Jones, B. F., \& Hanson, R. B. 1987, AJ, 94, 501

Law, D. R., Johnston, K. V., \& Majewski, S. R. 2005, ApJ, 619, 807

Lee, J.-F., \& van Altena, W. F. 1983, AJ, 88, 1683

Majewski, S. R. 1992, ApJS, 78, 87

Majewski, S. R., Law, D. R., Polak, A. A., \& Patterson, R. J. 2006, ApJ, 637, L25

Majewski, S. R., Skrutskie, M. F., Weinberg, M. D., \& Ostheimer, J. C. 2003, ApJ, 599, 1082

Majewski, S. R., et al. 2004, AJ, 128, 245

Martin, N. F., Ibata, R. A., Bellazzini, M., Irwin, M. J., Lewis, G. F., \& Dehnen, W. 2004, MNRAS, 348, 12
Martin, N. F., Irwin, M. J., Ibata, R. A., Conn, B. C., Lewis, G. F., Bellazzini, M., Chapman, S., \& Tanvir, N. 2006, MNRAS, 367, L69

Martínez-Delgado, D., Butler, D. J., Rix, H. W., Franco, Y. I., Peñarrubia, J., Alfaro, E. J., \& Dinescu, D. I. 2005, ApJ, 633, 205

Martínez-Delgado, D., Zinn, R., Carrera, R., \& Gallart, C. 2002, ApJ, 573, L19

Momany, Y., Zaggia, S., Gilmore, G., Piotto, G., Carraro, G., Bedin, L. R., \& De Angeli, F. 2006, A\&A, 451, 515

Monet, D. G., et al. 2003, AJ, 125, 984

Morrison, J. E., Roser, S., McLean, B., Bucciarelli, B., \& Laker, B. 2001, AJ, 121,1752

Munn, J. A., et al. 2004, AJ, 127, 3034

Newberg, H. J., et al. 2002, ApJ, 569, 245

Peñarrubia, J., et al. 2005, ApJ, 626, 128

Robin, A. C., Reylé, C., Derrière, S., \& Picaud, S. 2003, A\&A, 409, 523

Rocha-Pinto, H. J., Majewski, S. R., Skrutskie, M. F., \& Crane, J. D. 2003, ApJ, 594, L115

Rocha-Pinto, H. J., Majewski, S. R., Skrutskie, M. F., Crane, J. D., \& Patterson, R. J. 2004, ApJ, 615, 732

Rocha-Pinto, H. J., Majewski, S. R., Skrutskie, M. F., Patterson, R. J., Nakanishi, H., Muñoz, R. R., \& Sofue, Y. 2006, ApJ, 640, L147

Schlegel, D. J., Finkbeiner, D. P., \& Davis, M. 1998, ApJ, 500, 525

Schneider, D. P., et al. 2005, AJ, 130, 367

Seares, F. H., Kapteyn, J. C., \& van Rhijn, P. J. 1930, Mount Wilson Catalogue of Photographic Magnitudes in Selected Areas 1-139 (Washington: Carnegie Inst.)

Taff, L. G. 1981, Computational Spherical Astronomy (New York: Wiley)

Valdes, F. 1982, Proc. SPIE, 331, 465

Vivas, K. A., et al. 2004, AJ, 127, 1158

Yanny, B., et al. 2003, ApJ, 588, 824

. 2004, ApJ, 605, 575

Zacharias, N., Urban, S. E., Zacharias, M. I., Wycoff, G. L., Hall, D. M., Monet, D. G., \& Rafferty, T. J. 2004, AJ, 127, 3043 